\title{
Higgs production from sterile neutrinos at future lepton colliders
}

\author{
Stefan Antusch, ${ }^{a, b}$ Eros Cazzato $^{a}$ and Oliver Fischer ${ }^{a}$ \\ ${ }^{a}$ Department of Physics, University of Basel, \\ Klingelbergstr. 82, CH-4056 Basel, Switzerland \\ ${ }^{b}$ Max-Planck-Institut für Physik (Werner-Heisenberg-Institut), \\ Föhringer Ring 6, D-80805 München, Germany \\ E-mail: stefan.antusch@unibas.ch, e.cazzato@unibas.ch, \\ oliver.fischer@unibas.ch
}

ABSTRACT: In scenarios with sterile (right-handed) neutrinos that are subject to an approximate "lepton-number-like" symmetry, the heavy neutrinos (i.e. the mass eigenstates) can have masses around the electroweak scale and couple to the Higgs boson with, in principle, unsuppressed Yukawa couplings while accounting for the smallness of the light neutrinos' masses. In these scenarios, the on-shell production of heavy neutrinos and their subsequent decays into a light neutrino and a Higgs boson constitutes a hitherto unstudied resonant contribution to the Higgs production mechanism. We investigate the relevance of this resonant mono-Higgs production mechanism in leptonic collisions, including the present experimental constraints on the neutrino Yukawa couplings, and we determine the sensitivity of future lepton colliders to the heavy neutrinos. With Monte Carlo event sampling and a simulation of the detector response we find that, at future lepton colliders, neutrino Yukawa couplings below the percent level can lead to observable deviations from the SM and, furthermore, the sensitivity improves with higher center-of-mass energies (for identical integrated luminosities).

Keywords: Beyond Standard Model, Higgs Physics, Neutrino Physics

ARXIV EPRINT: 1512.06035 


\section{Contents}

1 Introduction 1

2 Sterile neutrinos at the electroweak scale $\quad 3$

2.1 The symmetry protected seesaw scenario 4

2.2 Weak interactions of the light and heavy neutrinos 5

2.3 Input parameters 6

3 Mono-Higgs production at future lepton colliders $\quad 7$

3.1 Mono-Higgs production in the SM 8

3.2 Non-unitarity effects in mono-Higgs production 9

$\begin{array}{lll}3.3 & \text { Resonant mono-Higgs production from sterile neutrinos decays } & 11\end{array}$

4 Simulation and analysis $\quad \mathbf{1 2}$

$\begin{array}{ll}4.1 \text { Analysis at the parton level } & 13\end{array}$

$\begin{array}{ll}\text { 4.1.1 Definition: signal, background, and significance } & 13\end{array}$

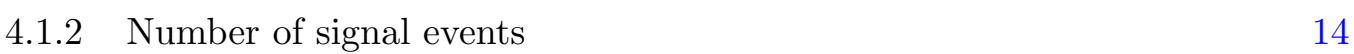

$\begin{array}{lll}\text { 4.1.3 Sensitivity to sterile neutrino parameters } & 14\end{array}$

$\begin{array}{lll}4.2 & \text { Reconstruction with the ILD detector } & 16\end{array}$

$\begin{array}{lll}\text { 4.2.1 Signal and background in the mono-Higgs channel } & 16\end{array}$

$\begin{array}{lll}\text { 4.2.2 Kinematic cuts } & 18\end{array}$

4.2.3 Future lepton collider sensitivity to the active-sterile mixing parameters 18

$\begin{array}{ll}\text { 4.2.4 Contamination of SM Higgs-boson parameters } & 19\end{array}$

$\begin{array}{llr}5 & \text { Summary and conclusions } & 20\end{array}$

$\begin{array}{ll}\text { A Cross sections and cuts } & 22\end{array}$

\section{Introduction}

Neutrino oscillation experiments have provided us with convincing evidence that (at least two of) the neutrinos are massive. More explicitly, for the three (active) neutrinos of the Standard Model (SM), two differences between the squared masses have been observed, i.e. $m_{2}^{2}-m_{1}^{2}=7.54_{-0.22}^{+0.26} \times 10^{-5} \mathrm{eV}^{2}$ and $\left|m_{3}^{2}-m_{1}^{2}\right|=(2.43 \pm 0.06) \times 10^{-3} \mathrm{eV}^{2}$ [1]. The values of the masses themselves cannot be measured via neutrino oscillations, but are bounded to lie below about $0.2 \mathrm{eV}$ from neutrinoless double beta experiments and cosmological constraints, see for instance ref. [2] for a recent review. With only the active neutrino degrees of freedom of the $\mathrm{SM}$, contained in the three $\mathrm{SU}(2)_{\mathrm{L}}$-lepton doublets, it is impossible to add a renormalizable term to the SM which accounts for the observed neutrino masses. 
However, renormalisable terms for neutrino masses can be introduced when righthanded (i.e. sterile) neutrinos are added to the field content of the SM. These sterile neutrinos are singlets under the gauge symmetries of the SM. They can have a so-called Majorana mass term, that involves exclusively the sterile neutrinos, as well as Yukawa couplings to the three active neutrinos from the $\mathrm{SU}(2)_{\mathrm{L}}$-lepton doublets and the Higgs doublet.

In the simplistic case, for only one active and one sterile neutrino, with a large Majorana mass $M$ and a Yukawa coupling $y$ such that $M \gg y v_{\mathrm{EW}}$, with $v_{\mathrm{EW}}$ the vacuum expectation value (vev) of the neutral component of the Higgs SU $(2)_{L}$-doublet, the mass of the light neutrino $m$ is simply given by $m \approx y^{2} v_{\mathrm{EW}}^{2} / M$, while the heavy state has a mass $\sim M$. The prospects for observing this type of sterile neutrino at collider experiments are not very promising: in order to explain the small mass of the light neutrinos (below, say, $0.2 \mathrm{eV}$ ), the mass of the heavy state would need to be of the order of the Grand Unification (GUT) scale for a Yukawa coupling of $\mathcal{O}(1)$, or, alternatively, the Yukawa coupling would be tiny, such that the active-sterile mixing would be highly suppressed.

In the more realistic case of three active neutrinos and several ${ }^{1}$ sterile neutrinos, however, the simple relation from above no longer holds and the possible values of the Majorana masses of the sterile neutrinos and the Yukawa couplings have to be reconsidered. In particular, if the theory entails for instance a "lepton-number-like" symmetry or a suitable discrete symmetry, sterile neutrinos with masses around the electroweak (EW) scale and unsuppressed (up to $\mathcal{O}(1)$ ) Yukawa couplings are theoretically allowed and the scenario is stable under radiative corrections. This scenario has the attractive features, that one does not have to introduce physics (much) above the EW scale - which avoids an explicit hierarchy problem - and one also does not have to introduce otherwise unmotivated tiny couplings. Various models of this type are known in the literature (see e.g. [3-8]). One example is the so-called "inverse seesaw" [3, 4], where the relation between light neutrino masses and sterile neutrinos masses is given by $m \approx \epsilon y^{2} v_{\mathrm{EW}}^{2} / M^{2}$, where $\epsilon$ is a small quantity that parametrizes the breaking of the protective symmetry. With $\epsilon$ controlling the magnitude of the light neutrino masses, for a given $M$ the coupling $y$ can in principle be large.

In this work, we base our studies on a benchmark scenario which captures the essential features of the realistic case, while it remains more general than specific models: the "symmetry protected seesaw scenario" (SPSS), that has also been discussed in ref. [9]. In this model, one pair of sterile neutrinos with a generic (approximate) protective symmetry is considered, where the two sterile neutrinos have opposite charges. Additional sterile neutrinos may exist, however it is assumed that their effects can be neglected as far as collider phenomenology is concerned. The parameters of the benchmark scenario that are relevant in the following are given by the mass parameter $M$, that defines the mass for the two heavy neutrinos due to the protective symmetry, and the moduli of the three Yukawa couplings $\left|y_{\nu_{e}}\right|,\left|y_{\nu_{\mu}}\right|$ and $\left|y_{\nu_{\tau}}\right|$ (or, equivalently, of the three active-sterile mixing angles, $\left.\left|\theta_{e}\right|,\left|\theta_{\mu}\right|,\left|\theta_{\tau}\right|\right)$. We focus on values of $M$ around the electroweak (EW) scale, which might be relevant for collider experiments. In ref. [9] the present constraints on the active-

\footnotetext{
${ }^{1}$ Since two mass differences in the oscillations of the light neutrinos were observed, at least two sterile neutrinos are required to give mass to at least two of the active neutrinos.
} 
sterile mixing for heavy neutrino masses above $\sim 10 \mathrm{GeV}$ have been calculated. Therein a combination of precision experiments was considered, which includes EW precision tests, lepton-flavour-violating decays at low energies ${ }^{2}$ (most strongly constrained by the results from the MEG collaboration [10]), tests of lepton universality, decays of the Higgs boson and direct searches at the large electron positron collider (LEP) by the collaborations Delphi [11], Opal [12], Aleph [13] and L3 [14]. Present and future constraints on EW scale sterile neutrinos have also been studied in the references in [15-36].

The present experimental bounds on the neutrino Yukawa couplings (and active-sterile mixings) are $\sim 5 \times 10^{-2}$ for heavy neutrinos in the considered mass range. These bounds allow for effects of the heavy neutrinos, which could be observed at future lepton colliders, such as the Future Circular Collider in the lepton mode (FCC-ee), the Circular Electron Positron Collider (CEPC) or the International Linear Collider (ILC). One of these effects is a production mechanism for the Higgs boson, which has first been considered in ref. [9]. In this mechanism the Higgs boson originates from the decay of a heavy neutrino, that has been produced on-shell, and is associated with two light neutrinos. This mechanism is referred to as resonant mono-Higgs production.

In this article, we study the mono-Higgs production mechanism in leptonic collisions ${ }^{3}$ for the center-of-energies of 240,350 , and $500 \mathrm{GeV}$. To be explicit, we consider the FCC-ee in the following and we expect the results to be representative for the CEPC and indicative for the ILC. In order to consolidate the previous estimate [9] for the sensitivity of the monoHiggs production cross section at $240 \mathrm{GeV}$ and to supplement the sensitivities at 350 and $500 \mathrm{GeV}$, we employ Monte Carlo event generators and simulate the detector response.

The paper is organized as follows: in section 2 we introduce the symmetry protected seesaw scenario. Section 3 contains a detailed description of the individual contributions to the mono-Higgs production mechanism from the SM and the heavy neutrinos. In the first part of section 4 we estimate the event counts and derive a parton-level sensitivity. In the second part of section 4 we extract a realistic sensitivity from a Monte Carlo event sample (including the SM background), including the simulation of the detector response. We discuss our results and conclude in section 5 .

\section{Sterile neutrinos at the electroweak scale}

As mentioned in the introduction, it is possible to have sterile (right-handed) neutrinos with masses around the electroweak (EW) scale and unsuppressed (up to $\mathcal{O}(1)$ ) Yukawa couplings, when a "lepton-number-like" symmetry is realized in the theory. The relevant features of seesaw models with such a protective symmetry may be represented in a benchmark scenario, which we refer to as the "symmetry protected seesaw scenario" (SPSS) (see also [9]) in the following.

\footnotetext{
${ }^{2}$ Note that lepton-flavour-violating decays probe the product of two active-sterile mixing angles, $\left|\theta_{\alpha}^{*} \theta_{\beta}\right|$, with $\alpha \neq \beta$.

${ }^{3}$ At the LHC these effects are suppressed by the larger QCD backgrounds and the reduced production cross section of the heavy neutrinos, see ref. [37] for a detailed analysis.
} 


\subsection{The symmetry protected seesaw scenario}

In the SPSS, we consider a pair of sterile neutrinos $N_{R}^{I}(I=1,2)$ and a suitable "leptonnumber-like" symmetry where $N_{R}^{1}\left(N_{R}^{2}\right)$ has the same (opposite) charge as the left-handed $\mathrm{SU}(2)_{L}$ doublets $L^{\alpha}, \alpha=e, \mu, \tau$. The masses of the light neutrinos and other (suppressed) lepton-number-violating effects arise, when this symmetry gets slightly broken. ${ }^{4}$ For the discussion of (lepton-number-conserving) signatures at lepton colliders, however, the effects from the small breaking of the protective symmetry will be neglected.

The Lagrangian density of a generic seesaw model with two sterile neutrinos in the symmetric limit is given by

$$
\mathscr{L} \supset \mathscr{L}_{\mathrm{SM}}-\overline{N_{R}^{1}} M N_{R}^{2 c}-y_{\nu_{\alpha}} \overline{N_{R}^{1}} \widetilde{\phi}^{\dagger} L^{\alpha}+\text { H.c. },
$$

where we omitted the kinetic terms of the sterile neutrinos, $\mathscr{L}_{\mathrm{SM}}$ contains the usual SM field content and with $L^{\alpha}$ and $\phi$ being the lepton and Higgs doublets, respectively. The $y_{\nu_{\alpha}}$ are the complex-valued neutrino Yukawa couplings and the sterile neutrino mass parameter $M$ can be chosen real without loss of generality.

Note that the benchmark scenario posits exactly two right-handed neutrinos, which we assume to be dominating the collider phenomenology. Furthermore, it captures the general features of symmetry protected seesaw scenarios with more than two right-handed neutrinos, provided that the effects of the additional right-handed neutrinos can be neglected. This can be the case, when the additional sterile neutrino(s) has large masses, or, alternatively, has zero charge under the "lepton-number-like" symmetry. In the limit of exact symmetry, the additional sterile neutrino(s) indeed decouples from the other particles, since no Yukawa couplings to the lepton doublets are allowed and they cannot mix with the other sterile states.

In the SPSS, the mass matrix of the two sterile neutrinos and the neutrino Yukawa matrix take the form

$$
M_{N}=\frac{1}{2}\left(\begin{array}{cc}
0 & M \\
M & 0
\end{array}\right), \quad Y_{\nu}=\left(\begin{array}{ll}
y_{\nu_{e}} & 0 \\
y_{\nu_{\mu}} & 0 \\
y_{\nu_{\tau}} & 0
\end{array}\right)
$$

where the zeroes correspond to the case of the "lepton-number-like" symmetry being exactly realised and are replaced with small quantities when the symmetry is slightly broken.

After EW symmetry breaking, we can write the $5 \times 5$ mass matrix of the electrically neutral leptons as:

$$
\mathscr{L}_{\text {mass }}=-\frac{1}{2}\left(\begin{array}{l}
\overline{\nu_{e_{L}}^{c}} \\
\frac{\nu_{\mu_{L}}^{c}}{\nu_{\tau_{L}}^{c}} \\
\frac{N_{R}^{1}}{N_{R}^{2}}
\end{array}\right)^{T}\left(\begin{array}{ccccc}
0 & 0 & 0 & m_{e} & 0 \\
0 & 0 & 0 & m_{\mu} & 0 \\
0 & 0 & 0 & m_{\tau} & 0 \\
m_{e} & m_{\mu} & m_{\tau} & 0 & M \\
0 & 0 & 0 & M & 0
\end{array}\right)\left(\begin{array}{c}
\nu_{e_{L}} \\
\nu_{\mu_{L}} \\
\nu_{\tau_{L}} \\
\left(N_{R}^{1}\right)^{c} \\
\left(N_{R}^{2}\right)^{c}
\end{array}\right)+\text { H.c. },
$$

\footnotetext{
${ }^{4}$ We remark that especially at the LHC, lepton-number-violating signatures can provide interesting search channels with low SM background, see e.g. refs. [38-45].
} 
with the Dirac masses $m_{\alpha}=y_{\nu_{\alpha}} v_{\mathrm{EW}} / \sqrt{2}$, and with $v_{\mathrm{EW}}=246.22 \mathrm{GeV}$. The diagonalisation of the mass matrix in eq. (2.3), referred to as $\mathcal{M}$ in the following, with a unitary matrix $U$, results in

$$
U^{T} \mathcal{M} U=\operatorname{Diag}(0,0,0, M, M),
$$

where $U$ is identified with the leptonic mixing matrix. In the symmetric limit, the three light neutrinos are massless and the two heavy neutrinos have degenerate mass eigenvalues. Note that correction of $\mathcal{O}\left(\theta^{2}\right)$ to the masses of the heavy neutrinos are neglected. Conversely, when the protective symmetry gets slightly broken, non-zero masses for two of the light neutrinos arise, and e.g. a third sterile neutrino could be added in order to explain a non-zero mass for the third light neutrino. The mixing of the active and sterile neutrinos can be quantified by the mixing angles, defined as

$$
\theta_{\alpha}=\frac{y_{\nu_{\alpha}}^{*}}{\sqrt{2}} \frac{v_{\mathrm{EW}}}{M} .
$$

With the leptonic mixing angles we can express the leptonic mixing matrix $U$ in eq. (2.4), in the limit of exact symmetry, as:

$$
U=\left(\begin{array}{ccccc}
\mathcal{N}_{e 1} & \mathcal{N}_{e 2} & \mathcal{N}_{e 3} & -\frac{\mathrm{i}}{\sqrt{2}} \theta_{e} & \frac{1}{\sqrt{2}} \theta_{e} \\
\mathcal{N}_{\mu 1} & \mathcal{N}_{\mu 2} & \mathcal{N}_{\mu 3} & -\frac{i}{\sqrt{2}} \theta_{\mu} & \frac{1}{\sqrt{2}} \theta_{\mu} \\
\mathcal{N}_{\tau 1} & \mathcal{N}_{\tau 2} & \mathcal{N}_{\tau 3} & -\frac{i}{\sqrt{2}} \theta_{\tau} & \frac{1}{\sqrt{2}} \theta_{\tau} \\
0 & 0 & 0 & \frac{i}{\sqrt{2}} & \frac{1}{\sqrt{2}} \\
-\theta_{e}^{*} & -\theta_{\mu}^{*} & -\theta_{\tau}^{*} & \frac{-i}{\sqrt{2}}\left(1-\frac{1}{2} \theta^{2}\right) & \frac{1}{\sqrt{2}}\left(1-\frac{1}{2} \theta^{2}\right)
\end{array}\right) .
$$

We remark that the leptonic mixing matrix, as shown above, is unitary up to second order in $\theta_{\alpha}$. The elements of the non-unitary $3 \times 3$ submatrix $\mathcal{N}$, which is the effective mixing matrix of the three active neutrinos, i.e. the Pontecorvo-Maki-Nakagawa-Sakata (PMNS) matrix, are given as

$$
\mathcal{N}_{\alpha i}=\left(\delta_{\alpha \beta}-\frac{1}{2} \theta_{\alpha} \theta_{\beta}^{*}\right)\left(U_{\ell}\right)_{\beta i},
$$

with $U_{\ell}$ being a unitary $3 \times 3$ matrix. Thus, in the limit of exact symmetry, the SPSS introduces seven additional parameters to the theory, the moduli of the neutrino Yukawa couplings $\left(\left|y_{\nu_{e}}\right|,\left|y_{\nu_{\mu}}\right|,\left|y_{\nu_{\tau}}\right|\right)$, their respective phase, and the sterile neutrino mass $M$, which can be studied in the context of collider phenomenology.

\subsection{Weak interactions of the light and heavy neutrinos}

Due to the mixing between the active and sterile neutrinos, the light and heavy neutrino mass eigenstates interact with the weak gauge bosons. The gauge interactions can be expressed by the currents of the neutral fermions in the mass basis, that are given by

$$
\begin{aligned}
j_{\mu}^{ \pm} & =\sum_{i=1}^{5} \sum_{\alpha=e, \mu, \tau} \frac{g}{\sqrt{2}} \bar{\ell}_{\alpha} \gamma_{\mu} P_{L} U_{\alpha i} \tilde{n}_{i}+\text { H.c. } \\
j_{\mu}^{0} & =\sum_{i, j=1}^{5} \sum_{\alpha=e, \mu, \tau} \frac{g}{2 c_{W}} \overline{\tilde{n}_{j}} U_{j \alpha}^{\dagger} \gamma_{\mu} P_{L} U_{\alpha i} \tilde{n}_{i},
\end{aligned}
$$


where $g$ is the weak coupling constant, $c_{W}$ is the cosine of the Weinberg angle and $P_{L}=$ $\frac{1}{2}\left(1-\gamma^{5}\right)$ is the left-chiral projection operator, and where the mass eigenstates $\tilde{n}_{j}$ of the active and sterile neutrinos are defined as

$$
\tilde{n}_{j}=\left(\nu_{1}, \nu_{2}, \nu_{3}, N_{4}, N_{5}\right)_{j}^{T}=U_{j \alpha}^{\dagger} n_{\alpha}, \quad n=\left(\nu_{e_{L}}, \nu_{\mu_{L}}, \nu_{\tau_{L}},\left(N_{R}^{1}\right)^{c},\left(N_{R}^{2}\right)^{c}\right)^{T} .
$$

Moreover, the neutrino mass eigenstates interact with the Higgs boson. The Yukawa terms in the mass basis, expanded up to $\mathcal{O}\left(\theta^{2}\right)$, can be expressed as

$$
\mathscr{L}_{\text {Yukawa }}=\frac{\sqrt{2} M}{v_{\text {EW }}}\left[\sum_{i=1}^{3}\left(\vartheta_{i 4}^{*} \overline{N_{4}^{c}}+\vartheta_{i 5}^{*} \overline{N_{5}^{c}}\right) \phi^{0} \nu_{i}+\sum_{j=4,5} \vartheta_{j j}^{*} \overline{N_{j}^{c}} \phi^{0} N_{j}\right]+\text { H.c. },
$$

with

$$
\vartheta_{i j}=\sum_{\alpha=e, \mu, \tau} U_{i \alpha}^{\dagger} U_{\alpha j}
$$

The partial decay widths of a sterile neutrino into weak gauge bosons and the Higgs boson, if kinematically allowed, are

$$
\begin{gathered}
\Gamma\left(N_{j} \rightarrow W^{ \pm} \ell_{\alpha}^{\mp}\right)=\frac{\left|\theta_{\alpha}\right|^{2}}{2} \frac{G_{F} M^{3}}{4 \sqrt{2} \pi} \Pi_{(1+1)}\left(\mu_{W}\right), \\
\Gamma\left(N_{j} \rightarrow Z \nu_{i}\right)=\left|\vartheta_{i j}\right|^{2} \frac{G_{F} M^{3}}{4 \sqrt{2} \pi} \Pi_{(1+1)}\left(\mu_{Z}\right), \\
\Gamma\left(N_{j} \rightarrow h \nu_{i}\right)=\left|\vartheta_{i j}\right|^{2} \frac{M^{3}}{8 \pi v_{\mathrm{EW}}^{2}}\left(1-\mu_{h}^{2}\right)^{2},
\end{gathered}
$$

where we introduced $\mu_{X}=m_{X} / M, G_{F}$ as the Fermi constant, and the kinematic factor

$$
\Pi_{(1+1)}\left(\mu_{X}\right)=\frac{1}{2}\left(1-\mu_{X}^{2}\right)^{2}\left(2+\mu_{X}^{2}\right) .
$$

For $M \gg m_{h}=125 \mathrm{GeV}$, the above partial decay widths result in branching ratios of the heavy neutrinos via $W: Z: H$ like $2: 1: 1$.

\subsection{Input parameters}

For the determination of the theory parameters, we use the set of input parameters with the highest experimental precision, i.e. the mass of the $Z$ boson, the fine structure constant (at the $Z$ pole) and the Fermi constant [1]. We note that the Fermi constant is inferred from the decays of the muon and interpreted in the context of the SM, such that we denote it by $G_{F}^{\mathrm{SM}}$ in the following.

\begin{tabular}{|l|c|c|c|}
\hline Input parameter & $m_{Z}[\mathrm{GeV}]$ & $\alpha\left(m_{Z}\right)^{-1}$ & $G_{F}^{\mathrm{SM}}\left[\mathrm{GeV}^{-2}\right]$ \\
\hline Value & $91.1875(21)$ & $127.944(14)$ & $1.1663787(6) \times 10^{-5}$ \\
\hline
\end{tabular}

In order to obtain the Fermi constant in the context of the SPSS, it can be related to $G_{F}^{\mathrm{SM}}$ by comparing the respective theory predictions for the muon decay cross sections. 
With the definition of the charged current interactions, according to eq. (2.8a), the cross section in the SPSS, for heavy neutrino masses $M \gg m_{\mu}$, is given by

$$
\sigma^{\mathrm{SPSS}}\left(\mu^{-} \rightarrow e^{-} \nu \bar{\nu}\right)=\left(\mathcal{N N}^{\dagger}\right)_{e e}\left(\mathcal{N N}^{\dagger}\right)_{\mu \mu} \times \sigma^{\mathrm{SM}}\left(\mu^{-} \rightarrow e^{-} \nu \bar{\nu}\right)
$$

where the summation over all possible final states is implied. Due to the non-unitarity of the PMNS matrix from eq. (2.7), the first factor on the right-hand side of the above equation is not equal to one, such that the cross sections for muon decay are different in both theories. The relation in eq. (2.14) between the cross sections fixes the relation of the Fermi constant in the context of the SPSS and the SM to (see e.g. [9, 46, 47])

$$
\left(G_{F}^{\mathrm{SM}}\right)^{2}=G_{F}^{2}\left(1-\left|\theta_{e}\right|^{2}\right)\left(1-\left|\theta_{\mu}\right|^{2}\right)
$$

This leads to a modification of the theory prediction for a number of other SM parameters, which will in the remainder of this paper be referred to as "non-unitarity effects". In particular, the theory prediction for the weak mixing angle $\theta_{W}$ (or, more commonly used, $\sin \theta_{W}$ ) at tree level (or in the on-shell scheme at any loop order) can be expressed as

$$
s_{W}^{2}=\frac{1}{2}\left[1-\sqrt{1-\frac{2 \sqrt{2} \alpha \pi}{G_{F}^{\mathrm{SM}} m_{Z}^{2}} \sqrt{\left(1-\left|\theta_{e}\right|^{2}\right)\left(1-\left|\theta_{\mu}\right|^{2}\right)}}\right] .
$$

From the relation $m_{Z}^{2} c_{W}^{2}=m_{W}^{2}$, we obtain the modified prediction for the $W$ boson mass. Furthermore, the vev of the Higgs boson in the SPSS is given by

$$
v_{\mathrm{EW}}=\frac{1}{\sqrt{\sqrt{2} G_{F}}}=246.22\left[1-0.25\left(\left|\theta_{e}\right|^{2}+\left|\theta_{\mu}\right|^{2}\right)\right] .
$$

A more detailed discussion and up-to-date constraints on the model parameters can be found in [9].

\section{Mono-Higgs production at future lepton colliders}

We refer to an event as mono Higgs, when it contains exclusively (the decay products of) a Higgs boson and a significant amount of missing energy. In the SPSS the missing energy is due to the light neutrinos escaping detection. We note that the heavy neutrinos decay inside the detector volume for the considered active-sterile mixings and masses. In this section we study the effects of sterile neutrinos on the cross section for mono-Higgs production in the context of future lepton colliders, i.e. the process

$$
e^{+} e^{-} \rightarrow h \bar{\nu} \nu
$$

Generally, in the SPSS we can split the total cross section for this process into the following three contributions

$$
\sigma_{h \nu \nu}=\sigma_{h \nu \nu}^{\mathrm{SM}}+\sigma_{h \nu \nu}^{\mathrm{Non}-\mathrm{U}}+\sigma_{h \nu \nu}^{\text {Direct }} .
$$




\begin{tabular}{|c|ccc|}
\hline$\sqrt{s}$ & $240 \mathrm{GeV}$ & $350 \mathrm{GeV}$ & $500 \mathrm{GeV}$ \\
\hline Experiments & FCC-ee, CEPC & FCC-ee (CEPC, ILC) & FCC-ee (ILC) \\
Luminosity/year & $3.5 \mathrm{ab}^{-1}$ & $1.0 \mathrm{ab}^{-1}$ & $0.3 \mathrm{ab}^{-1}$ \\
years & 3 & 3.5 & 3 \\
\hline
\end{tabular}

Table 1. Different center-of-mass energies, with currently discussed target integrated luminosity for the FCC-ee [48, 50], that are also representative to some extent for the CEPC [49]. The ILC is included in parentheses, because it is foreseen to operate with polarised beams, which is not considered in the following.

The first contribution is the expression for mono-Higgs production in the SM, with the two main mechanisms for Higgs production given by Higgs strahlung and $W W$ fusion. The second contribution contains exclusively the non-unitarity effects which modify the low-energy input parameters as well mixing of the active neutrinos. The third contribution includes the direct production of Higgs bosons from the decays of heavy neutrinos. The remainder of this section is dedicated to the study of these contributions, up to second order in the active-sterile mixing angles in the context of the considered future lepton collider options.

We focus on the center-of-mass energies $\sqrt{s}=240,350 \mathrm{GeV}$, that are being discussed for the FCC-ee [48] (and the CEPC [49]) to study the properties of the Higgs boson and top quark, respectively. We will also include $\sqrt{s}=500 \mathrm{GeV}$, which can be reached according to present discussion by the FCC-ee working group, see e.g. ref. [50]. We remark, that the considered center-of-mass energies together with integrated luminosities of order $\mathrm{ab}^{-1}$ can also be achieved by the ILC [51]. However, since the linear colliders are considering polarised beams, we limit the discussion in the following to the circular machines. The relevant machine performance parameters are listed in table 1.

\subsection{Mono-Higgs production in the SM}

At $e^{+} e^{-}$colliders, the most important SM-Higgs-production mechanisms are Higgs strahlung, $e^{+} e^{-} \rightarrow Z^{*} \rightarrow Z h$ and $W W$ fusion, $e^{+} e^{-} \rightarrow h \bar{\nu}_{e} \nu_{e}$, respectively. The fraction of the $Z$ decays into neutrinos constitutes the Higgs strahlung contribution to the mono-Higgs signature. Notice that in $W W$ fusion only electron neutrinos are produced, (since there is no flavour mixing in the SM,) contrary to Higgs strahlung, where all neutrino flavours are produced equally. The Feynman diagrams for the two mono-Higgs-production mechanisms are shown in figure 1, where we omit the display of explicit indices of the final state neutrinos.

The contribution to the cross section for mono-Higgs production from Higgs strahlung can be expressed in the narrow width approximation as

$$
\sigma_{h \nu \nu}^{H Z}:=\sigma^{\mathrm{SM}}\left(e^{+} e^{-} \rightarrow h Z\right) \times \operatorname{Br}(Z \rightarrow \nu \bar{\nu}),
$$

where the branching ratio $\operatorname{Br}(Z \rightarrow \nu \bar{\nu})$ is set to $20.0 \%$ and we implicitly summed over all combinations of final states. The SM Higgs strahlung cross section is given by [52]

$$
\sigma^{\mathrm{SM}}\left(e^{+} e^{-} \rightarrow h Z\right)=\frac{G_{f}^{2} m_{Z}^{4}}{24 \pi}\left(v_{e}^{2}+a_{e}^{2}\right) \lambda^{\frac{1}{2}} \frac{\lambda s+12 m_{Z}^{2}}{\left(s-m_{Z}^{2}\right)^{2}},
$$



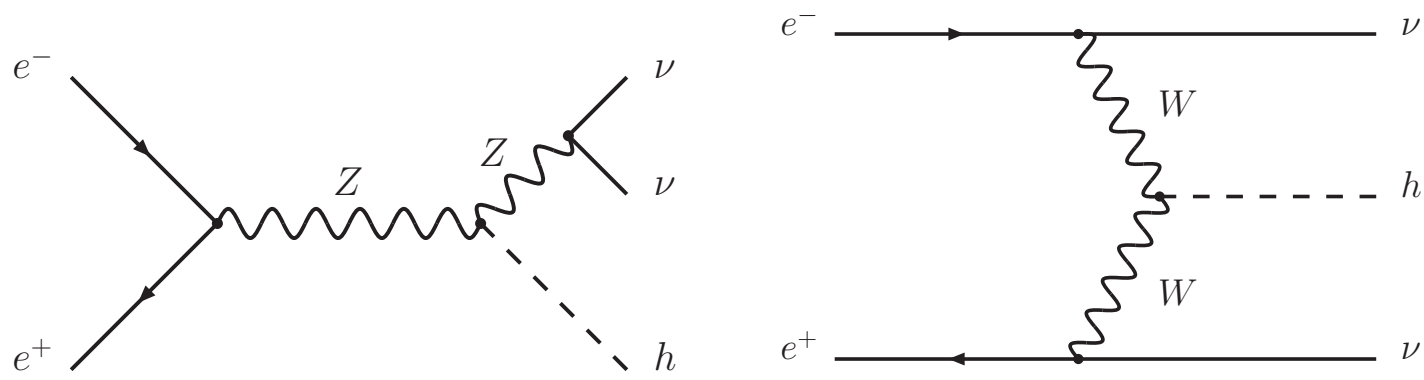

Figure 1. The main mechanisms for Higgs boson production plus missing energy in the SM. The Higgs boson is produced by Higgs strahlung or WW fusion.

with the center-of-mass energy $\sqrt{s}$, the axial- and vector-coupling of the electron-current to the $Z$ boson $a_{e}=-1 / 2$ and $v_{e}=-1 / 2+2 s_{W}^{2}$, and the phase-space factor

$$
\lambda=\left(1-\frac{\left(m_{h}+m_{Z}\right)^{2}}{s}\right)\left(1-\frac{\left(m_{h}-m_{Z}\right)^{2}}{s}\right) .
$$

The contribution to the cross section for mono-Higgs production from $W W$ fusion is $[52]$

$$
\sigma_{h \nu \nu}^{W W}:=\frac{G_{f}^{3} m_{W}^{4}}{4 \sqrt{2} \pi^{3}} \Pi_{h \nu \nu}
$$

with the phase space factor

$$
\begin{aligned}
\Pi_{h \nu \nu} & =\int_{x_{h}}^{1} d x \int_{x}^{1} \frac{d y F(x, y)}{\left(1+(y-x) / x_{W}\right)^{2}}, \\
F(x, y) & =\left(\frac{2 x}{y^{3}}-\frac{1+3 x}{y^{2}}+\frac{2+x}{y}-1\right)\left(\frac{z}{1+z}-\log [1+z]\right)+\frac{x}{y^{3}} \frac{z^{2}(1-y)}{1+z},
\end{aligned}
$$

where $x_{h}=m_{h}^{2} / s, x_{W}=m_{W}^{2} / s$ and $z=y\left(x-x_{h}\right) /\left(x x_{W}\right)$.

The mono-Higgs-production cross section $\sigma_{h \nu \nu}^{S M}$ is given by the sum of $\sigma_{h \nu \nu}^{W W}, \sigma_{h \nu \nu}^{H Z}$ and a contributing interference term. We show in figure 2 the individual contributions to the mono-Higgs-production cross section, their naive sum, and the total cross section. For the sake of simplicity, we neglect the interference term in the following discussion. This is a good approximation since at 240 and $350 \mathrm{GeV}$ it contributes less than $5 \%$ to the total cross section, which we have checked numerically with WHIZARD, and with the formulae from ref. [52]. We emphasize, however, that in our analysis we use the full expression for $\sigma_{h \nu \nu}^{S M}$. As we can see from figure 2 , the cross section for mono-Higgs production at $\sqrt{s}=240 \mathrm{GeV}$ is dominated by the contribution from Higgs strahlung, contrary to $\sqrt{s}=500 \mathrm{GeV}$, where the contribution from $W W$ fusion has taken over.

\subsection{Non-unitarity effects in mono-Higgs production}

In this section, we discuss how the effects from the modified properties of the light (mostly active) neutrinos lead to a deviation of the mono-Higgs-production cross section from the 


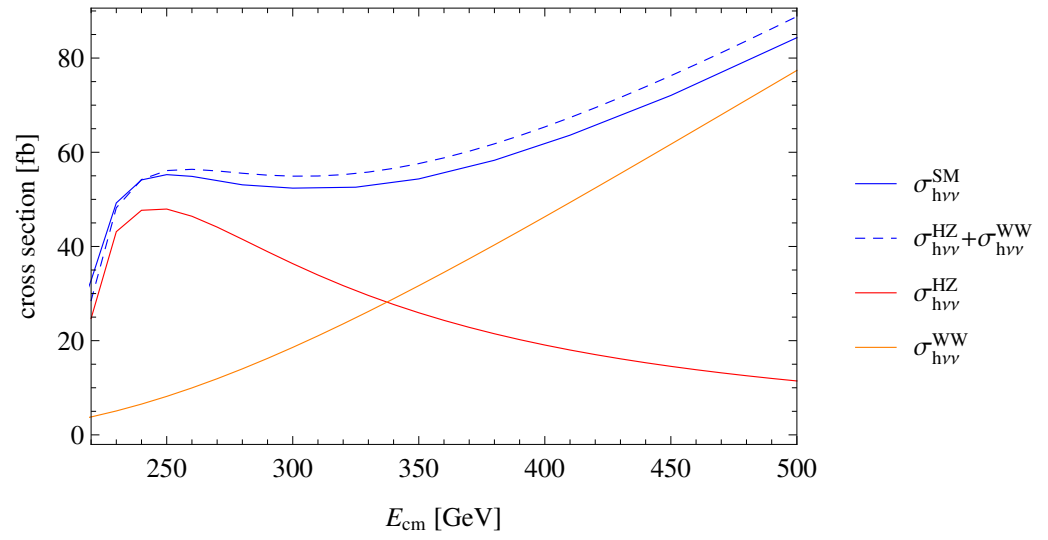

Figure 2. The total mono-Higgs-production cross section and the individual contributions from Higgs strahlung $\left(\sigma_{h \nu \nu}^{H Z}\right)$ and from $W W$ fusion $\left(\sigma_{h \nu \nu}^{W W}\right)$, respectively. The dashed blue line denotes the naive sum of $\sigma_{h \nu \nu}^{H Z}$ and $\sigma_{h \nu \nu}^{W W}$, with the small interference term neglected, see text for details.

SM prediction. This modification manifests itself in the non-unitarity of the effective PMNS matrix $\mathcal{N}$ which we refer to as the non-unitarity effects. Note that these effects do not include exchange, nor production and decay, of the heavy neutrinos.

One part of the non-unitarity effects stem from the modification of the input parameters, as described in section 2.3. In particular, the dependence of the Fermi constant on the active-sterile mixing parameters, cf. eq. (2.15), introduces a global change in the definitions for $\sigma_{h \nu \nu}^{H Z}$ and $\sigma_{h \nu \nu}^{W W}$, see eqs. (3.4) and (3.6). Also the electroweak parameters $\sin \theta_{W}$ and $m_{W}$ add to the effect.

The other part of the non-unitarity effects comes from the modification of the vertices according to eq. (2.8), where the non-unitary PMNS matrix enters. The partial monoHiggs-production cross section $\sigma_{h \nu \nu}^{H Z}$ is therefore proportional to $\left|\sum_{i, j=1,2,3}\left(\mathcal{N}^{\dagger} \mathcal{N}\right)_{i j}\right|^{2}$, whereas $\sigma_{h \nu \nu}^{W W}$ is proportional to $\left|\sum_{i, j=1,2,3}\left(\mathcal{N}_{j e}^{\dagger} \mathcal{N}_{e i}\right)\right|^{2}$, where the flavour index " $e$ " is fixed by the incident lepton beams. Notice that it is possible to have two different light neutrinos in the final state, i.e. $i \neq j$.

We combine the above discussed non-unitarity effects and expand in the small activesterile mixing parameters to order $\theta^{2}$, so that we can write the deviation from the SM predicted mono-Higgs-production cross section as:

$$
\sigma_{h \nu \nu}^{\mathrm{Non}-\mathrm{U}}=\sigma_{h \nu \nu}^{\mathrm{SM}} \sum_{\alpha=e, \mu, \tau} c_{\alpha}(\sqrt{s})\left|\theta_{\alpha}\right|^{2}+\mathcal{O}\left(\theta^{4}\right) .
$$

The coefficients $c_{\alpha}$ are dependent on the center-of-mass energy: firstly, the relative contribution from Higgs strahlung and $W W$ fusion to the total cross section varies with $\sqrt{s}$, and, secondly, both diagrams vary differently with the active-sterile mixing parameters. In table 2 we list the resulting numerical values of the coefficients for the center-of-mass energies 240, 350 and $500 \mathrm{GeV}$.

For $\left|\theta_{\tau}\right|$ substantially smaller than $\left|\theta_{e, \mu}\right|$, the deviation in the cross section due to nonunitarity is positive, contrary to the case of dominating $\left|\theta_{\tau}\right|$, where the negative coefficient 


\begin{tabular}{|c|ccc|}
\hline$\sqrt{s} / \mathrm{GeV}$ & 240 & 350 & 500 \\
\hline$c_{e}$ & 0.88 & 0.26 & 0.10 \\
$c_{\mu}$ & 1.08 & 1.28 & 1.70 \\
$c_{\tau}$ & -0.53 & -0.40 & -0.05 \\
\hline
\end{tabular}

Table 2. List of the coefficients from eq. (3.8) obtained with WHIZARD 2.2.7 [53, 54]. The numerical precision of the coefficients is $\pm 0.04, \pm 0.05$ and \pm 0.05 at the center-of-mass energy of 240,350 and $500 \mathrm{GeV}$, respectively.
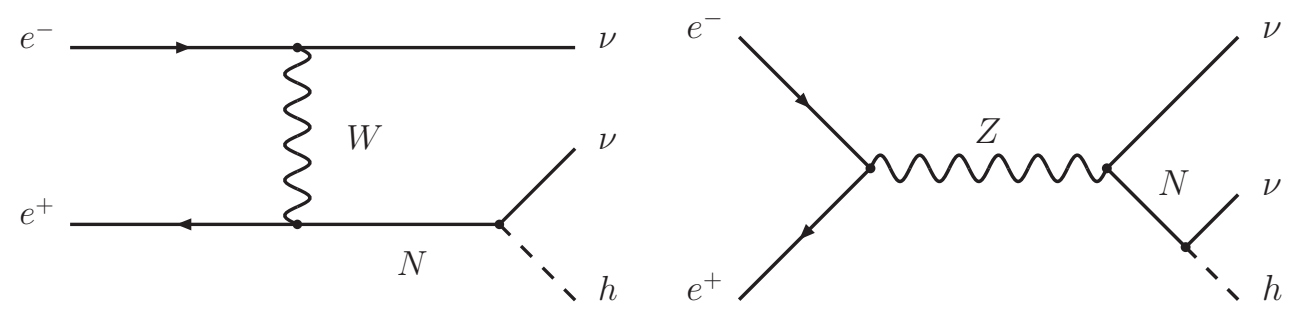

Figure 3. The two dominating Feynman diagrams that give rise to the partial mono-Higgs production cross section $\sigma_{h \nu \nu}^{\text {Direct }}$ involving the exchange of heavy neutrinos and leading to a resonant enhancement of the mono-Higgs production.

$c_{\tau}$ in eq. (3.8) leads to a negative deviation in the cross section which is formally given by a negative $\sigma_{h \nu \nu}^{\mathrm{Non}-\mathrm{U}}$.

\subsection{Resonant mono-Higgs production from sterile neutrinos decays}

The last contribution to the mono-Higgs-production cross section, $\sigma_{h \nu \nu}^{\text {Direct }}$, includes the effects from the exchange of virtual heavy neutrinos, see figure 3 , for the corresponding Feynman diagrams. We note that the contribution from the s-channel Higgs-exchange diagram to the production of heavy neutrinos is neglected, due to the smallness of the electron Yukawa coupling. This diagram might become relevant when considering muon colliders. The on-shell production and subsequent decay of a heavy neutrino into a Higgs boson and a light neutrino, yields a resonantly enhanced contribution to the mono-Higgs production.

The expression $\sigma_{h \nu \nu}^{\text {Direct }}$ also includes the interference between the amplitudes stemming from the Feynman diagrams in figure 1, and those from the diagrams in figure 3. It turns out that the interference of these two sets of amplitudes is negligible, because one part is proportional to the small ratios $\frac{m_{e}^{2}}{s}$ and $\frac{m_{\nu}^{2}}{s}$, and the other part, resembling the contribution of the Majorana mass of the heavy neutrinos, is cancelled out by the protective symmetry. We therefore write to a very good approximation

$$
\sigma_{h \nu \nu}^{\text {Direct }}=\sum_{i, j, k} \sigma\left(e^{+} e^{-} \rightarrow N_{j} \nu_{i}\right) \times \operatorname{Br}\left(N_{j} \rightarrow h \nu_{k}\right)+\mathcal{O}\left(\theta^{4}\right),
$$

with the branching ratios for the heavy neutrinos derived from eqs. (2.12), and the production cross section $\sigma\left(e^{+} e^{-} \rightarrow N_{j} \nu_{i}\right)$, that can be found for instance in ref. [55]. We show $\sigma_{h \nu \nu}^{\text {Direct }}$ as a function of the heavy neutrino mass $M$ for four different center-of-mass energies in the two panels of figure 4, using eq. (3.9). In order to illustrate the effects of the 

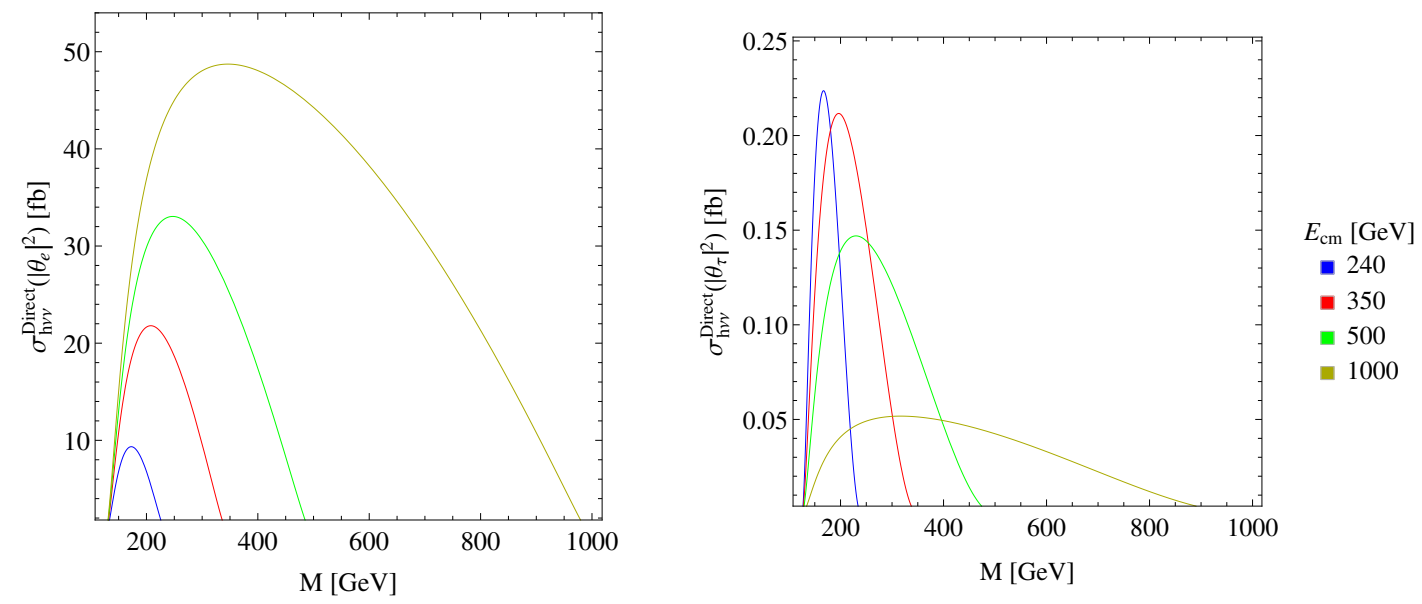

Figure 4. $\sigma_{h \nu \nu}^{\text {Direct }}$ as a function of the heavy neutrino mass. Left: the active-sterile mixing parameter $\left|\theta_{e}\right|^{2}=0.0018$ is chosen to saturate the $1 \sigma$ upper bound from ref. [9], while $\left|\theta_{\mu, \tau}\right|=0$ are used. Right: active-sterile mixing parameter $\left|\theta_{\tau}\right|^{2}=0.0042$, which saturates the $1 \sigma$ upper bound, while $\left|\theta_{e, \mu}\right|=0$. In this figure, the formula from ref. [55] has been used for $\sigma\left(e^{+} e^{-} \rightarrow \nu N\right)$.

different $\theta_{\alpha}$, in the left panel of the figure we use the values $\left|\theta_{e}\right|^{2}=0.0018,\left|\theta_{\mu}\right|=\left|\theta_{\tau}\right|=0$ and in the right panel the values $\left|\theta_{\tau}\right|^{2}=0.0042,\left|\theta_{e}\right|=\left|\theta_{\mu}\right|=0$. Both sets of example values are within the $1 \sigma$ upper bound given in ref. [47].

Some remarks on figure 4 are in order at this point. The right panel shows the contribution to the production cross section coming exclusively from the s-channel exchange of a $Z$ boson, cf. the right diagram in figure 3, which is proportional to $\sum_{\alpha}\left|\theta_{\alpha}\right|^{2}$. Notice, that the production cross section decreases with increasing center-of-mass energy, since this contribution is suppressed $\sim \frac{1}{s}$ for $s>m_{Z}$.

The left panel receives contributions from the exchange of both, the $Z$ and the $W$ boson, and for the considered center-of-mass energies, it is dominated by the latter, cf. the left diagram in figure 3. Comparing the magnitudes of $\sigma_{h \nu \nu}^{\text {Direct }}$ from the left panel with $\sigma_{h \nu \nu}^{\mathrm{SM}}$ in figure 2 it is evident that the resonant contribution from heavy neutrinos can indeed be sizeable, and becomes more relevant at higher energies. Therefore, for center-of-mass energies of $240 \mathrm{GeV}$ or higher, this results in $\sigma_{h \nu \nu}^{\text {Direct }}$ being mostly sensitive to $\left|\theta_{e}\right|$, since the vertex of the $W$ boson with the heavy sterile neutrino and the electron is proportional to the matrix element $U_{14}$ or respectively $U_{15}$ of the leptonic mixing matrix, cf. eq. (2.6), with both matrix elements being proportional to $\left|\theta_{e}\right|$. We remark that a muon collider (see e.g. [56]) would allow to test the parameter $\left|\theta_{\mu}\right|$ with great precision and sterile neutrinos with large masses.

\section{Simulation and analysis}

In this section we quantify the contribution from the decays of sterile neutrinos (cf. diagrams in figure 3), which is considered to constitute the signal for our analysis, over the SM background (corresponding to the diagrams in figure 1) through an analysis of Monte Carlo generated event samples. We first analyse the sterile neutrino effects at the parton level, and then include also the simulation of the detector response. In our analysis we consider pro- 
cesses at the tree level, which is sufficient since in our scenario the one-loop level effects are negligible (cf. [57]). Furthermore, we include effects up to order $\theta^{2}$ in the active-sterile mixing parameters. In order to extract the flavour information from the neutrino Yukawa couplings, we use the present constraints from [9] and analyse the effect of each one individually.

Three cases. As discussed above, the SPSS has four parameters that are relevant for our considerations: $\left|y_{\nu_{e}}\right|,\left|y_{\nu_{\mu}}\right|,\left|y_{\nu_{\tau}}\right|$ and the sterile neutrino mass $M$. In the following, to investigate the effects of the Yukawa couplings separately, we will consider the three limiting cases where only one of them is non-zero:

$$
\begin{array}{rll}
\text { Case I: effects from }\left|y_{\nu_{e}}\right| \leftrightarrow y_{\nu_{e}} \neq 0, & y_{\nu_{\mu}}=0, & y_{\nu_{\tau}}=0, \\
\text { Case II: effects from }\left|y_{\nu_{\mu}}\right| \leftrightarrow y_{\nu_{e}}=0, & y_{\nu_{\mu}} \neq 0, & y_{\nu_{\tau}}=0, \\
\text { Case III: effects from }\left|y_{\nu_{\tau}}\right| \leftrightarrow y_{\nu_{e}}=0, & y_{\nu_{\mu}}=0, & y_{\nu_{\tau}} \neq 0 .
\end{array}
$$

Present constraints on the sterile neutrino parameters. The constraints have recently been calculated in $[9,47]$, based on a large set of observables, including e.g. the present bounds on EW precision observables, universality test, lepton flavour violating charged lepton decays and the direct searches for neutral leptons at LEP. For heavy neutrino masses in the range $m_{Z} \leq M \leq \sqrt{s}$, with $\sqrt{s}=240,350$ and $500 \mathrm{GeV}$, the constraints can be expressed as upper bounds on the neutrino Yukawa couplings, which, at the $1 \sigma$ Bayesian confidence level, can be approximated by:

$$
\left|y_{\nu_{e}}\right|=0.042 \frac{\sqrt{2} M}{v_{\mathrm{EW}}}, \quad\left|y_{\nu_{\mu}}\right|=0.015 \frac{\sqrt{2} M}{v_{\mathrm{EW}}}, \quad\left|y_{\nu_{\tau}}\right|=0.065 \frac{\sqrt{2} M}{v_{\mathrm{EW}}} .
$$

\subsection{Analysis at the parton level}

As a first step, we consider the contribution from sterile neutrinos to the mono-Higgs production at the parton level. This analysis allows us to establish an order-of-magnitude estimate for the sensitivity of this process to the neutrino Yukawa couplings, and the deviation from the SM prediction. In order to generate the event distributions at the parton level, we implemented the sterile neutrino (SPSS) benchmark model via Feynrules [58] into the Monte Carlo event generator Madgraph5_aMC@NLO [59] and analysed the output with madanalysis5 [60].

\subsubsection{Definition: signal, background, and significance}

At the parton level, the investigated final state is given by a Higgs boson and two neutrinos, i.e. Higgs boson plus missing energy. We define the signal of our analysis to be given by the events that are produced via resonant mono-Higgs production from sterile neutrinos, see section 3.3, together with the events stemming from the non-unitarity contribution in section 3.2. The number of signal events is thus given by $N_{S}=\left|\sigma_{h \nu \nu}-\sigma_{h \nu \nu}^{\mathrm{SM}}\right| \times \mathcal{L}$, with the integrated luminosity $\mathcal{L}$ according to table 1 . Notice that due to the indirect effect from the input parameters $\sigma_{h \nu \nu}$ may be smaller than $\sigma_{h \nu \nu}^{\mathrm{SM}}$ in case III. We define the background by the events that stem from Higgs strahlung and $W W$ fusion in the SM (i.e. with active-sterile mixing set to zero), as discussed in section 3.1. The number of SM predicted background events is therefore simply given by $N_{B}=\sigma_{h \nu \nu}^{\mathrm{SM}} \times \mathcal{L}$. 
In order to quantify differences between the two models, we define the significance:

$$
\mathcal{S}=\frac{N_{S}}{\sqrt{N_{B}+N_{S}}}
$$

The denominator corresponds to the statistical standard deviation of the total number of events, which is equivalent to the $1 \sigma$ standard deviation when normal distributions are assumed. ${ }^{5}$ The above defined significance therefore measures the difference in event counts between the SM and the SPSS in units of standard deviations.

\subsubsection{Number of signal events}

We estimate the number of mono-Higgs-produced signal events $N_{S}$, stemming from each flavour corresponding to the cases I,II and III of eq. (4.1), that are compatible with the present upper bounds on $\left|y_{\nu_{e}}\right|,\left|y_{\nu_{\mu}}\right|$ and $\left|y_{\nu_{\tau}}\right|$ from eq. (4.2) for the FCC-ee. The number of signal events were calculated from the Madgraph5_aMC@NLO-generated cross sections for eight values of $M$ at $240 \mathrm{GeV}$ and nine values of $M$ for each, 350 and $500 \mathrm{GeV}$. They typically lead to an excess over the number of background events and are shown, together with the number of background events, in figure 5, for the considered center-of-mass energies: in the figure, the blue and red lines show the results for the cases I and II, respectively, where the parameters $\left|y_{\nu_{e}}\right|$ and $\left|y_{\nu_{\mu}}\right|$ are non-zero. For case III, with non-zero $\left|y_{\nu_{\tau}}\right|$, a partial cancellation between non-unitarity effects and direct production of heavy neutrinos occurs. The situation, where $\sigma_{h \nu \nu}<\sigma_{h \nu \nu}^{\mathrm{SM}}$ is shown by the dashed green line, while $\sigma_{h \nu \nu}>\sigma_{h \nu \nu}^{\mathrm{SM}}$ is denoted by the solid green line. The number of SM background events is shown as a solid black line. The dashed black line corresponds to $\sqrt{N_{B}} \approx \sqrt{N_{B}+N_{S}}$ for the considered event numbers.

As figure 5 shows, up to $\mathcal{O}\left(10^{5}\right)$ signal events on the parton level can be produced for the machine parameters from table 1 , when non-zero $\left|y_{\nu_{e}}\right|$ compatible with its present bounds is considered. Comparing this to the SM predicted number of background events $N_{B}$ we see that the contribution of the heavy neutrinos to the mono-Higgs-production cross section can be sizeable. As anticipated in the previous section, $\left|y_{\nu_{e}}\right|$ has by far the largest impact on the mono-Higgs production cross section.

\subsubsection{Sensitivity to sterile neutrino parameters}

We now turn to the possible sensitivity of the mono-Higgs channel at future lepton colliders (cf. table 1) to the neutrino Yukawa coupling $\left|y_{\nu_{e}}\right|$ (respectively the active-sterile mixing parameter $\left.\left|\theta_{e}\right|\right)$ for a given $M$. The sensitivity is defined as the value of $\left|y_{\nu_{e}}\right|$ that corresponds to a significance of $\mathcal{S}=1$ (cf. eq. (4.3)), i.e. to a signal at the $1 \sigma$ level. $^{6}$ Notice, that the sensitivity to the neutrino Yukawa couplings $y_{\nu_{\mu}}$ and $y_{\nu_{\tau}}$ does not improve the present bounds, which is why we omit to discuss the sensitivity for case II and III. We show the resulting sensitivity to the modulus of the neutrino Yukawa coupling $y_{\nu_{e}}$ in figure 6 . In the

\footnotetext{
${ }^{5}$ The number of events is Poisson distributed which, for the large expected event numbers, approaches the normal distribution.

${ }^{6}$ Note that $\mathcal{S}=1$ corresponds to $84 \%$ confidence level for a one-sided normal distribution, which is chosen here such that the results derived in the following can directly be compared with the corresponding limits in ref. [9].
} 

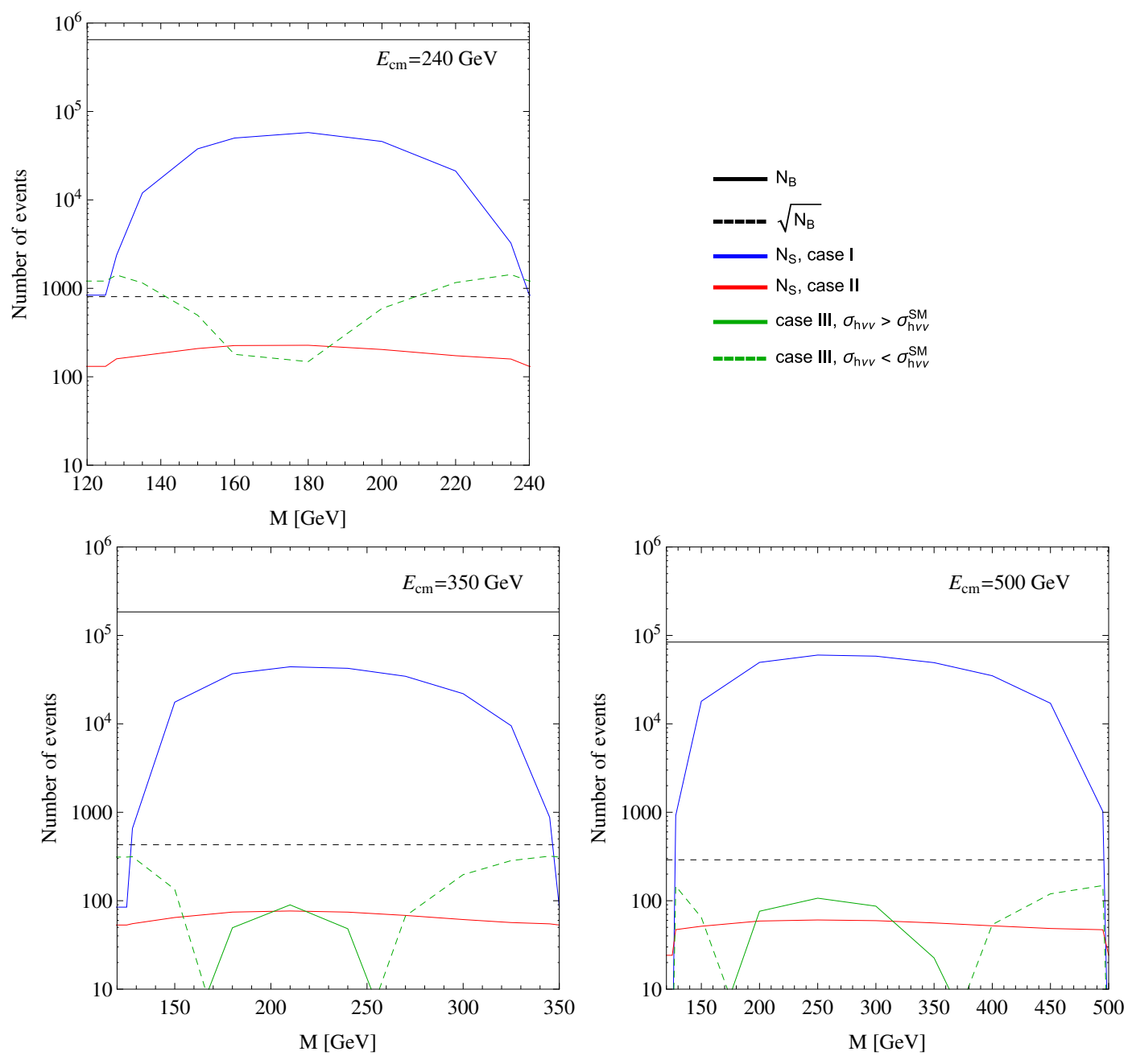

Figure 5. Number of signal events $N_{S}$ in mono-Higgs production at the parton level for the three cases as defined in eq. (4.1), with Yukawa coupling values compatible with present $1 \sigma$ bounds from refs. $[9,47]$. The solid black lines denote the number of background events $N_{B}$, and the dashed black lines denote $\sqrt{N_{B}}$. The machine performance parameters are specified in table 1 .

figure, the red, blue and green line corresponds to the sensitivities for $\sqrt{s}=240,350$ and $500 \mathrm{GeV}$, respectively, and the black dashed line denotes the present constraints from [9]. We have simulated $10^{6}$ background events and the same number of events for eight values of $M$ for $\sqrt{s}=240 \mathrm{GeV}$, and nine values of $M$ for each, $\sqrt{s}=350$ and $500 \mathrm{GeV}$. For each simulation, we have optimised the cuts to obtain highest sensitivity. Those cuts can be obtained straightforwardly from the distribution of the Higgs boson momentum at the parton level and a selection is shown in table 8 .

Remarkably, apart from probing a wider mass range, the sensitivity to $\left|y_{\nu_{e}}\right|$ at $350 \mathrm{GeV}$ is comparable to the center-of-mass energy of $240 \mathrm{GeV}$, despite the lower integrated luminosity. The same is true for $500 \mathrm{GeV}$, where $1 \mathrm{ab}^{-1}$ can lead to comparable sensitivities to 350 and $240 \mathrm{GeV}$ even for $M \sim 200 \mathrm{GeV}$, which is due to the evolution of $\sigma_{h \nu \nu}^{\mathrm{SM}}$ and $\sigma_{h \nu \nu}^{\text {Direct }}$ with the center-of-mass energy. Furthermore it is worth noting that for $M<m_{h}$ and $\sqrt{s}<M$ the sensitivity stems from non-unitarity effects, i.e. the indirect effects from 


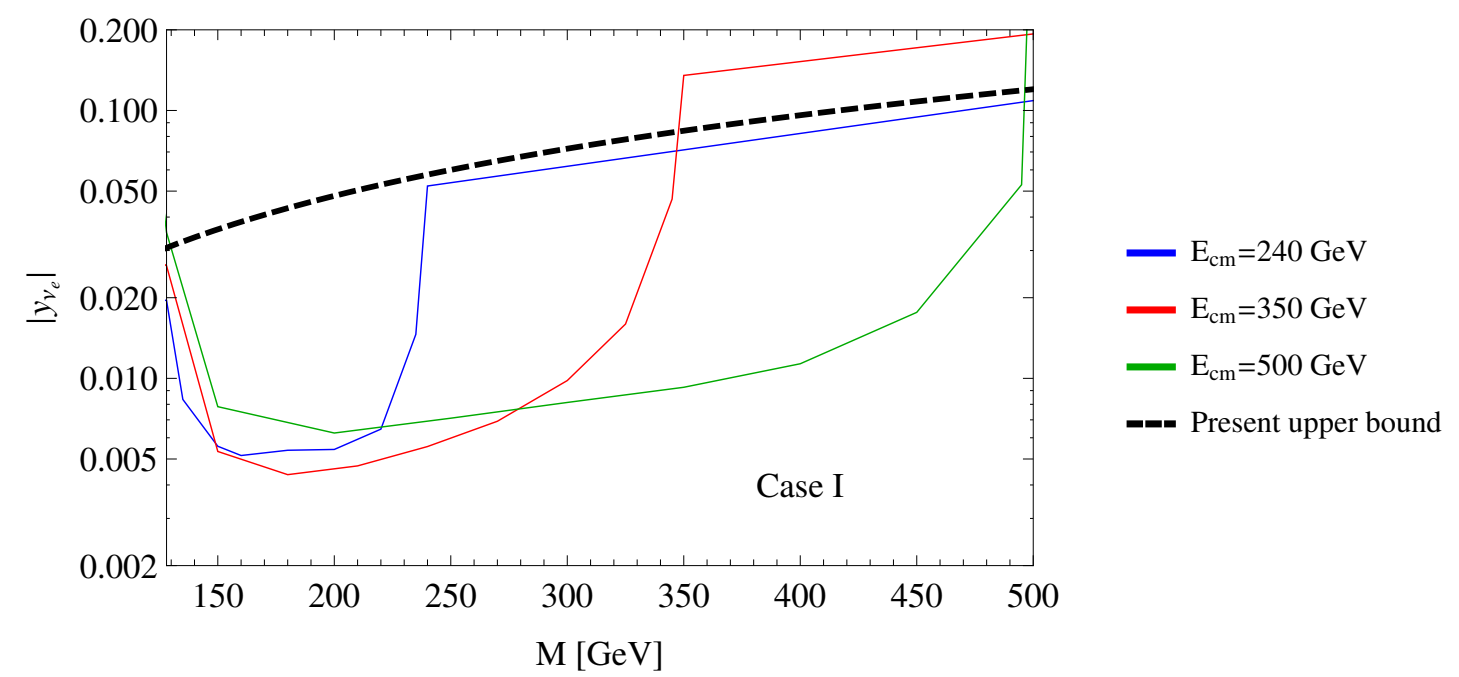

Figure 6. Sensitivity of the mono-Higgs production cross section to the neutrino Yukawa couplings at the parton level at $1 \sigma$, with the machine performance parameters from table 1 .

active-sterile mixing on the input parameters and the modified interactions of the light neutrinos. In this case the number of signal events is given by eq. (3.8). For $\sqrt{s}=240 \mathrm{GeV}$ we find that even for $M<m_{h}$ and $\sqrt{s}<M$, the present constraints on $y_{\nu_{e}}$ allow for a $\sim 1 \sigma$ deviation from the SM prediction for the mono-Higgs-production cross section.

\subsection{Reconstruction with the ILD detector}

In this section we describe the relevant SM background and how to extract the mono-Higgs candidates from the reconstructed events after the simulation of the detector response. From those mono-Higgs candidate events we calculate a more realistic sensitivity of the mono-Higgs channel to the neutrino Yukawa coupling $y_{\nu_{e}}$. Furthermore, we show that the resonant mono-Higgs production can also lead to a contamination of the mono-Higgs candidate event sample, when "standard cuts" are applied.

For the analysis we have generated the signal and background with the Monte Carlo event generator WHIZARD 2.2.7 [53, 54], which allows for the appropriate simulation of leptonic collisions including initial state radiation. We remark that the effects from beamstrahlung are negligible for the considered center-of-mass energies and will be neglected in the following. The parton showering and hadronisation has been carried out with PYTHIA 6.427 [61] and the events were reconstructed with the ILD detector card using Delphes 3.2.0 [62].

\subsubsection{Signal and background in the mono-Higgs channel}

For the analysis at the reconstructed level, the parton level final states have to be transformed into reconstructed objects. In particular, the light neutrinos manifest themselves as missing energy, and the Higgs bosons decay into $b \bar{b}(57.7 \%), W W^{*}(21.6 \%), g g(8.50 \%)$, $\tau^{+} \tau^{-}(6.37 \%), c \bar{c}(2.66 \%)$, and $Z Z^{*}(2.46 \%)$. Higgs boson candidates can be reconstructed from its decay products, which have an invariant mass around $m_{h}$. In order to obtain better statistics for resonantly produced mono-Higgs events from heavy neutrino decays, we focus on the Higgs decays into two hadronic jets (di-jet) which have a very large combined 
branching ratio of $\sim 70 \%$. The di-jet plus missing energy signature comprises our monoHiggs search channel such that we select events with two hadronic jets with an invariant mass of $100 \mathrm{GeV} \leq M_{j j} \leq 140 \mathrm{GeV}$.

The signal is here given by events that stem from the decays of the heavy neutrinos, which add to the number of events in the search channel. When considering inclusive processes on the reconstructed level, two mechanisms involving heavy neutrinos contribute to the signal: the resonant mono-Higgs and the resonant mono- $\mathrm{Z}$ production mechanisms, where the latter is defined analogously to the former, with the $\mathrm{Z}$ originating from the decay of a heavy neutrino. However, the invariant mass of the resonant mono- $Z$ produced di-jet is $\sim m_{Z}$, such that the above defined cuts for the mono-Higgs search channel essentially remove this contribution from the signal. We remark that the resonant mono- $\mathrm{Z}$ production constitutes an independent search channel for the heavy neutrinos, and provides an important consistency check for this model, because the relative amount of additional (resonantly produced) events at the Higgs and $\mathrm{Z}$ pole, respectively, is predicted by the model parameters. A detailed study of this channel is beyond the scope of this paper and is therefore left for future work.

For the SM background, we include all processes with a four fermion final state, that can be (mis-)identified as a mono-Higgs-candidate event. We do not consider processes with di-electrons or di-muons in the final state as background, because it is very unlikely to misidentify two light leptons as a jet at the same time.

The dominating background is given by $q \bar{q} \nu \nu$, with $q=b, c, g$ stemming from monoHiggs production in the SM, cf. section 3. In addition to the mono-Higgs production process, we find the subdominant background consists in processes with $q \bar{q} \nu \nu$ final states, where the quarks $q=b, c, s, d, u$ are produced via gauge bosons and in radiative processes. We note that, due to our selection criterion of the invariant di-jet mass being around $m_{h}$, most of the backgrounds that stem from gauge boson decays are efficiently suppressed to below the percent level. Other subdominant backgrounds come from final states with four hadronic jets, $e^{+} e^{-}$plus di-jet, $2 \tau$-jets plus di-jet, and single-top and $t \bar{t}$ final states, when kinematically allowed.

We simulate and reconstruct $10^{5}$ events for each final state, with the exception for dib-jet plus missing energy, where $3 \times 10^{6}$ events have been simulated and reconstructed. We note that we simulate inclusive processes such that the interference between the possible production mechanisms is accounted for. A detailed list of the included backgrounds and their corresponding cross sections is given in table 4 in the appendix.

For illustrative purposes we show the di-jet invariant mass distribution in the monoHiggs search channel in figure 7. Therein the center-of-mass energy is set to $240 \mathrm{GeV}$ and the model parameters are set to $M=152 \mathrm{GeV}$ and $\left|y_{\nu_{e}}\right|=0.036$, which saturates the present upper bounds at $1 \sigma$. The dominant and subdominant background is represented by the red and green area, respectively. The figure shows how resonant mono-Higgs production, represented by the blue area, contributes substantially to the SM predicted number of events. We remark that the Higgs peak is located at the invariant di-jet mass $\sim 120 \mathrm{GeV}$ instead of at the Higgs boson mass $m_{h}=125 \mathrm{GeV}$. This shift is due to an energy loss of the hadronic jet, due to the emission of light neutrinos during hadronisation. This has no further implication for our analysis. 


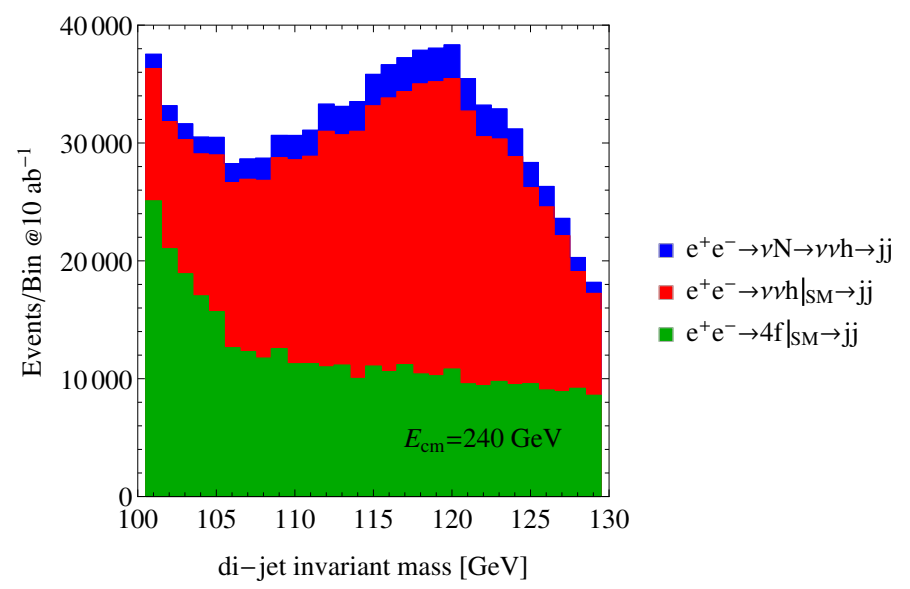

Figure 7. The di-jet invariant mass spectrum in the mono-Higgs search channel $(j j$ plus missing energy) after simulation of the ILD detector response at $E_{c m}=240 \mathrm{GeV}$. The red and green area denote the dominant and subdominant background, respectively, see text for further details. The blue area denotes the signal from a heavy neutrino with a mass of $152 \mathrm{GeV}$, and a Yukawa coupling to the electron flavour, $y_{\nu_{e}}$, saturating the present upper bounds from precision data [9, 47].

\subsubsection{Kinematic cuts}

For the analysis in the following, we first select the mono-Higgs search channel by applying the above defined selection criteria. After this pre-selection we study the kinematic distributions of the di-jet momentum $\left(P_{j j}\right)$, the missing transverse momentum $\left(E_{T}\right)$, the angular separation of the two jets, and the momentum and energy of the individual jets. We find that the most efficient observable to enhance the significance of the signal, cf. eq. (4.3), is given by $P_{j j}$. Furthermore, the $E_{T}$ is very powerful in removing the non-mono-Higgs SM background at $\sqrt{s}=240 \mathrm{GeV}$. A detailed list of the applied cuts and the resulting efficiencies can be found in tables 5, 6 and 7 in the appendix.

A comment on b-tagging is in order at this point. We find that, with a nominal selection efficiency of $\sim 0.7$ for a b-flavoured heavy jet, the resulting sensitivity at $240 \mathrm{GeV}$ does not improve the one derived from blindly accepting all hadronic jets. We therefore neglect b-tagging, which may become relevant when a more sophisticated kinematic analysis is applied.

\subsubsection{Future lepton collider sensitivity to the active-sterile mixing parameters}

To establish the sensitivity of the mono-Higgs search channel at future lepton colliders to the active-sterile neutrino mixing, we use the definition from section 4.1.3 for a significance of $1 \sigma$. In order to enhance the sensitivity, we have employed a series of kinematic cuts that are listed in tables 5, 6 and 7 in the appendix, together with the resulting numbers of signal and background events. The resulting sensitivities to the neutrino Yukawa coupling $\left|y_{\nu_{e}}\right|$ for the considered center-of-mass energies are shown in figure 8 for several values of the heavy neutrino mass:

The figure shows that, despite the increased background after reconstruction, the resulting sensitivities from the parton level (denoted by the dotted lines) and the recon- 


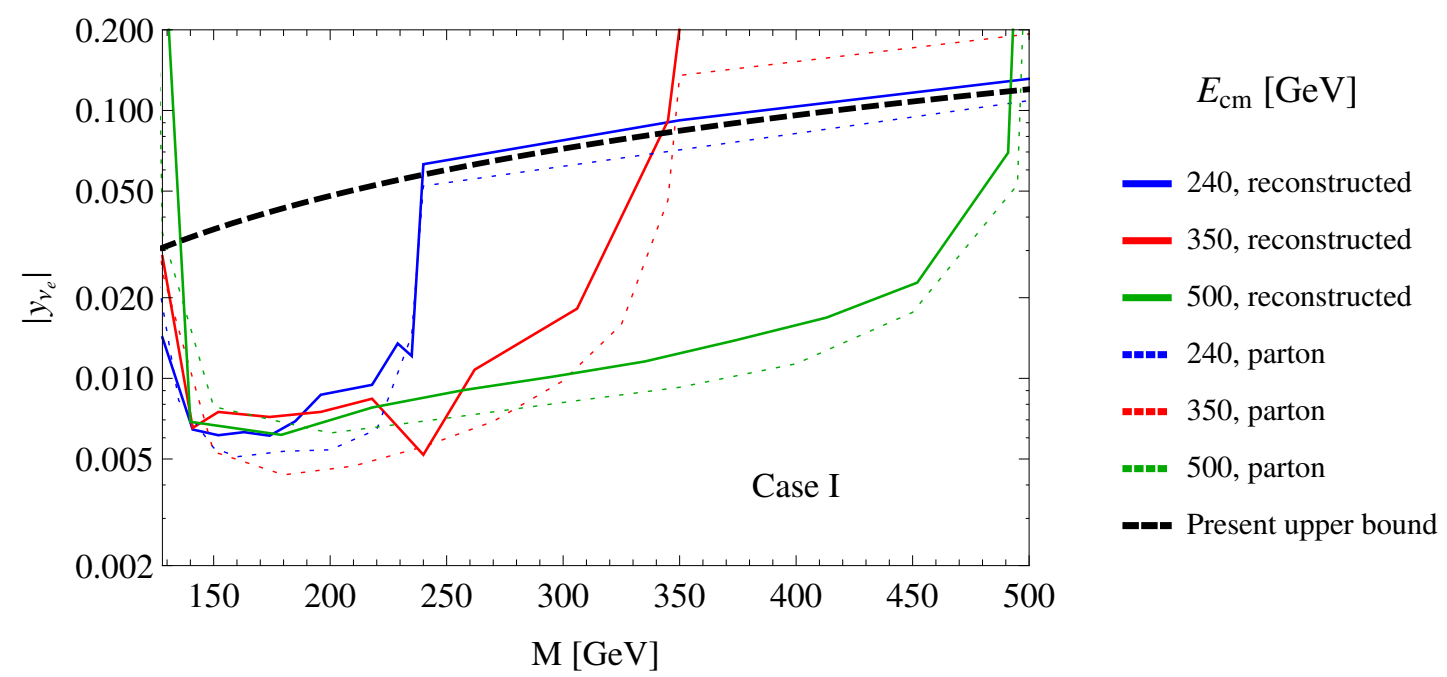

Figure 8. Future lepton collider sensitivity of the mono-Higgs search channel, i.e. di-jet plus missing energy signature, to the neutrino Yukawa coupling $y_{\nu_{e}}$ at $1 \sigma$. We use the machine performance parameters from table 1 , simulate the response of the ILD detector and apply kinematic cuts according to tables 5,6 and 7 .

structed level are comparable in magnitude. ${ }^{7}$ Values of the neutrino Yukawa coupling $y_{\nu_{e}}$ above the solid lines give rise to a signal that can be distinguished from the SM background with a significance larger than $1 \sigma$. The sensitivity at $500 \mathrm{GeV}$ is competitive with 240 and $350 \mathrm{GeV}$, even for heavy neutrino masses $M \leq 250 \mathrm{GeV}$ and despite the lower luminosity. Moreover, for values of the heavy neutrino mass above the kinematic threshold (i.e. $M>\sqrt{s}$ ), the signal is due to the non-unitarity effects in mono-Higgs production (cf. section 3.2), however the corresponding sensitivity is weaker than the present bound.

We remark that resonant mono-Higgs production can give rise to events with larger amount of missing energy compared to the SM. This provides an unambiguous signal without SM background. However, the considered target luminosities results in $\mathcal{O}(1)$ and $\mathcal{O}$ (10) signal events at 240 and $350 \mathrm{GeV}$, respectively, such that they do not provide an improvement of the sensitivity. We note, that at $500 \mathrm{GeV}$, the considered luminosity results in less than $\mathcal{O}(1)$ events of this kind.

Altogether the FCC-ee shows a remarkable sensitivity to the electron neutrino Yukawa coupling which leads to very promising prospects for discovering heavy neutrino signals in the mono-Higgs channel.

\subsubsection{Contamination of SM Higgs-boson parameters}

For the analysis of the Higgs boson at future lepton colliders so-called "standard cuts" have been defined ${ }^{8}$ in [63], which we show in table 3. Those cuts are designed to improve the ratio of mono-Higgs events over SM background events. However in the case of resonant mono-Higgs production they turn out not to be as efficient in filtering out the additional events from heavy neutrino decays, as is shown in tables 5 and 6 . This contamination of the

\footnotetext{
${ }^{7}$ It may be possible to further improve on the cuts, up to the point where the sensitivity on the reconstructed level is identical to, or even better than the parton level sensitivity, which we leave for future work.

${ }^{8}$ We thank F. Müller for assistance with the "standard cuts" for the extraction of mono-Higgs events at lepton colliders.
} 


\begin{tabular}{|l|cc|}
\hline$\sqrt{s}$ & $240 \mathrm{GeV}$ & $350 \mathrm{GeV}$ \\
\hline Missing Mass $[\mathrm{GeV}]$ & $80 \leq M_{\text {miss }} \leq 140$ & $50 \leq M_{\text {miss }} \leq 240$ \\
Transverse P $[\mathrm{GeV}]$ & $20 \leq P_{T} \leq 70$ & $10 \leq P_{T} \leq 140$ \\
Longitudinal $\mathrm{P}[\mathrm{GeV}]$ & $\left|P_{L}\right|<60$ & $\left|P_{L}\right|<130$ \\
Maximum P $[\mathrm{GeV}]$ & $|P|<30$ & $|P|<60$ \\
Di-jet Mass $[\mathrm{GeV}]$ & $100 \leq M_{j j} \leq 130$ & $100 \leq M_{j j} \leq 130$ \\
Angle (jets) $[\mathrm{Rad}]$ & $\alpha>1.38$ & $\alpha>1.38$ \\
\hline
\end{tabular}

Table 3. "Standard cuts" from ref. [63] to optimize the ratio of mono-Higgs signal to SM background for future lepton colliders. The angle $\alpha$ refers to the opening angle formed by the two hadronic jets.

sample of mono-Higgs events can lead to a deviation from the theory prediction, for instance in the mono-Higgs production cross section, when interpreted in the context of the SM. We remark that no "standard cuts" for $500 \mathrm{GeV}$ exist, and it has only been considered for the FCC-ee very recently. Thus, even though this center-of-mass energy constitutes an excellent environment for studying the mono-Higgs channel, we do not include it in the following.

In figure 9 we show the ensuing deviation of the contaminated mono-Higgs production cross section from the SM prediction, for $\left|y_{\nu_{e}}\right|$ saturating the present upper bound. The statistical accuracy at $1 \sigma$ are denoted by the black and grey dashed lines for 240 and $350 \mathrm{GeV}$, respectively. The figure shows that the deviation of the mono-Higgs production cross section can be significant compared to the experimental accuracy. This can lead to a discrepancy when comparing the Higgs properties derived from the contaminated monoHiggs sample with the other Higgs channels at $240 \mathrm{GeV}$ [49]. Moreover, up to a $3 \sigma$ deviation from the SM predicted mono-Higgs production cross section is possible at $240 \mathrm{GeV}$ and at $350 \mathrm{GeV}$ the deviation can be larger than $5 \sigma$.

We emphasise that the shown deviation of the mono-Higgs-production cross section from the SM prediction is fully compatible with present constraints on the active-sterile mixing. Furthermore, if the present non-zero best-fit value for $\left|\theta_{e}\right|$ as reported in refs. [9, $47,64]$ get confirmed, an observable deviation in the number of mono-Higgs events would be a prediction.

\section{$5 \quad$ Summary and conclusions}

In this work we have studied Higgs production from sterile neutrinos at future lepton colliders. We have considered a scenario with a pair of sterile (right-handed) neutrinos that are subject to an approximate "lepton-number-like" symmetry. In this scenario the heavy neutrinos (i.e. the mass eigenstates) can have masses around the electroweak scale and couple to the Higgs boson with, in principle, unsuppressed Yukawa couplings while accounting for the smallness of the light neutrinos' masses. We refer to this as the "symmetry protected seesaw scenario" (SPSS).

The sterile neutrinos in the SPSS enable a novel Higgs production mechanism, given by the on-shell production of a heavy neutrino and its subsequent decay into a light neutrino and a Higgs boson: resonant mono-Higgs production. Due to the comparatively large neutrino Yukawa couplings inducing large active-sterile neutrino mixings, the heavy neutrinos 


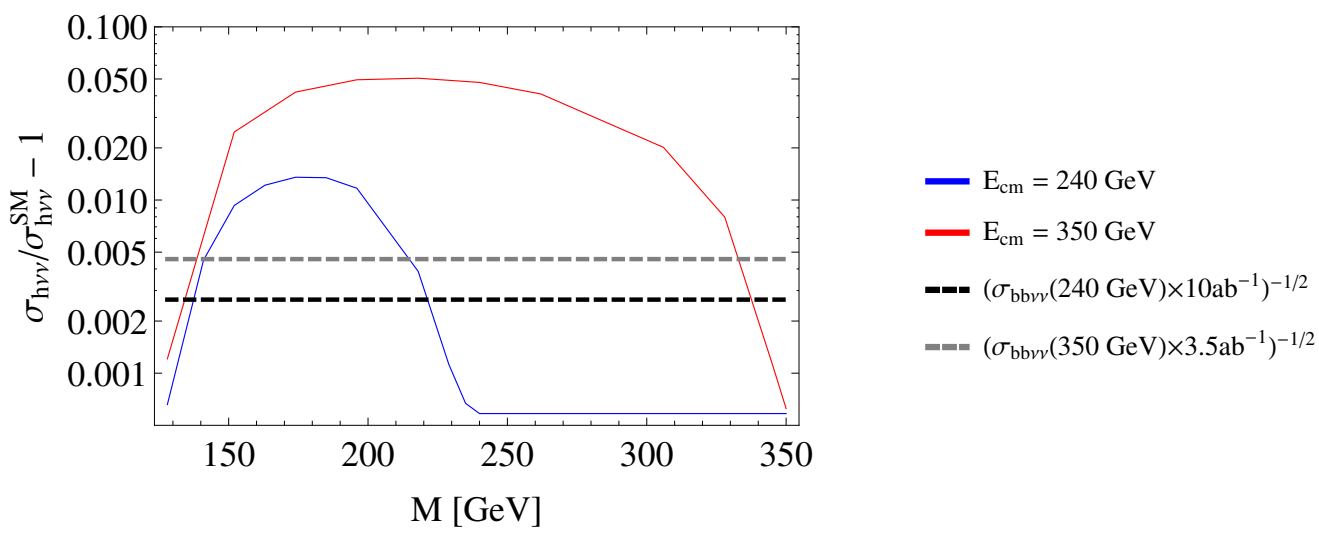

Figure 9. Heavy neutrino induced deviation of the mono-Higgs production cross section when "standard cuts" are applied (cf. table 3) to the contaminated event sample when $y_{\nu_{e}}$ saturates the $1 \sigma$ upper bound. The horizontal dashed lines denote the relative statistical precision of the SM predicted events $N$, given by $1 / \sqrt{N}$.

can be produced efficiently from lepton collisions. Therefore, future lepton colliders provide a promising environment for testing heavy neutrinos, including signals from resonant mono-Higgs production.

On the contrary, in hadronic collisions the heavy neutrinos can only be produced from the decays of a weak gauge boson, which results in a strong suppression of the heavyneutrino-production cross section. In addition there are large QCD backgrounds, and only transversal projections of the kinematic observables can be studied. Altogether, the sensitivity to the resonant mono-Higgs production at the LHC is much weaker than at the considered future lepton colliders (see ref. [37]).

In order to assess the prospects for testing resonant mono-Higgs production at future lepton colliders, we consider the FCC-ee in the following and we expect the results to be representative for the CEPC and indicative for the ILC.

For $\sqrt{s}$ we consider $240 \mathrm{GeV}, 350 \mathrm{GeV}$ and $500 \mathrm{GeV}$ as currently discussed in the working groups. We have generated Monte Carlo event samples for the SM background and the heavy neutrino signal, where we used the present $1 \sigma$ upper bounds for the active-sterile mixing parameters and we simulated the detector response.

We find that the number of resonantly produced mono-Higgs events can be as large as $\sim 10 \%$ of the SM predicted number at $240 \mathrm{GeV}$. Furthermore, the upper bound on the resonant mono-Higgs events can be up to $\sim 30 \%$ and $\sim 40 \%$ at $\sqrt{s}$ of 350 and $500 \mathrm{GeV}$, respectively. Via the mono-Higgs channel, the FCC-ee would be sensitive to the neutrino Yukawa coupling $\left|y_{\nu_{e}}\right|$ (respectively to the active-sterile mixing parameter $\left|\theta_{e}\right|$ ) down to $\sim 5 \times 10^{-3}$ (cf. figure 8). Interestingly, higher $\sqrt{s}$ not only allows for an increased range of testable heavy neutrino masses $M$, but also the signal-to-background ratio increases such that a comparable sensitivity can be achieved with less integrated luminosity.

Moreover, we have shown that the resonantly produced mono-Higgs events can effectively contaminate the SM analysis of the mono-Higgs channel, as shown in figure 9 . With $\left|\theta_{e}\right|$ within the present $1 \sigma$ upper bounds, this can lead to deviations from the SM prediction at the percent level, much larger than the estimated future accuracy. 
In summary, we discussed a novel channel for Higgs production, namely resonant mono-Higgs production from sterile neutrinos. It can induce sizeable deviations from the SM mono-Higgs prediction and can be used as a sensitive probe of sterile neutrino properties at lepton colliders.

\section{Acknowledgments}

This work has been supported by the Swiss National Science Foundation. We thank Lorenzo Basso for assisting with Madgraph5_aMC@NLO and madanalysis5, for valuable discussions, and for reading the manuscript. We furthermore thank Jürgen Reuter for assisting with WHIZARD and Michele Selvaggi for helping with the ILD card for DELPHES. O.F. is thankful for stimulating discussions at the first FCC-ee mini-Higgs-Workshop at CERN.

\section{A Cross sections and cuts}

\begin{tabular}{|l|c|c|c|}
\hline Final state & $\sigma^{\mathrm{SM} @ 240 \mathrm{GeV}[\mathrm{fb}]}$ & $\sigma^{\mathrm{SM} @ 350 \mathrm{GeV}[\mathrm{fb}]}$ & $\sigma^{\mathrm{SM} @ 500 \mathrm{GeV}[\mathrm{fb}]}$ \\
\hline$b \bar{b} \nu \nu$ & 146.492 & 134.614 & 183.594 \\
$c \bar{c} \nu \nu$ & 88.0172 & 73.7956 & 82.7041 \\
$j j \nu \nu$ & 528.8 & 463.1 & 500.3 \\
$b \bar{b} b \bar{b}$ & 81.2629 & 47.6152 & 25.5571 \\
$b \bar{b} c \bar{c}$ & 146.566 & 87.6518 & 51.6446 \\
$b \bar{b} j j$ & 6820.6 & 4259.5 & 2537.8 \\
$b \bar{b} e^{+} e^{-}$ & 2080.87 & 2500.82 & 2920.9 \\
$b \bar{b} \tau^{+} \tau^{-}$ & 34.1905 & 19.7975 & 11.0619 \\
$c \bar{c} \tau^{+} \tau^{-}$ & 25.2553 & 15.0695 & 9.15227 \\
$j j \tau^{+} \tau^{-}$ & 116.0 & 72.4 & 37.6 \\
$\tau^{+} \tau^{-} \nu \nu$ & 235.89 & 163.851 & 119.989 \\
$\operatorname{single~top~}$ & 0.012 & 63.3 & 1092 \\
$t \bar{t}$ & - & 322. & 574. \\
\hline
\end{tabular}

Table 4. Included Standard Model four fermion background to the mono-Higgs channel, for details see text. We separated hadronic jets from heavy (charm and bottom) quarks, and denote events with light jets from up, down, strange quarks, and gluons with a $j$. The cross sections for both tables have been evaluated with WHIZARD 2.2.7 [53, 54], the efficiency was obtained with the cuts from table 3 via madanalysis5 [60]. 


\begin{tabular}{|c|c|c|c|c|}
\hline$M[\mathrm{GeV}]$ & $P_{j j}[\mathrm{GeV}]$ & $N_{S}$ & $N_{B}$ & $N_{S}^{\mathrm{SM}}$ \\
\hline 128 & $>80$ & 308 & 4287 & 25.1 \\
141 & $>70$ & 3780 & 18627 & 1327 \\
152 & $>70$ & 4846 & 18627 & 2951 \\
163 & $>70$ & 5286 & 18627 & 3924 \\
174 & $>60$ & 8759 & 34946 & 4387 \\
185 & $>70$ & 5652 & 18627 & 4358 \\
196 & $>80$ & 1935 & 4287 & 3762 \\
218 & $>70$ & 4192 & 18637 & 1113 \\
229 & $>75$ & 1505 & 8147 & 182 \\
235 & $>75$ & 1966 & 8147 & 29 \\
\hline
\end{tabular}

Table 5. List of kinematic cuts for the extraction of the sensitivity in figure 8. For all benchmark points for the heavy neutrino mass $M$ at $\sqrt{s}=240 \mathrm{GeV}$, the cuts $110 \mathrm{GeV} \leq M_{j j} \leq 125 \mathrm{GeV}$ and $E_{T}>15 \mathrm{GeV}$ have been applied. The number of SM background events, $N_{B}^{\mathrm{SM}}$, after application of the "standard cuts" is 338600 .

\begin{tabular}{|c|c|c|c|c|c|c|}
\hline$M[\mathrm{GeV}]$ & $M_{j j}[\mathrm{GeV}]$ & $P_{j j}[\mathrm{GeV}]$ & $E_{T}[\mathrm{GeV}]$ & $N_{S}$ & $N_{B}$ & $N_{S}^{\mathrm{SM}}$ \\
\hline 128 & $100-130$ & $100-170$ & - & 384 & 109908 & 210 \\
141 & $110-125$ & $70-160$ & - & 3581 & 17695 & 8652 \\
152 & $110-125$ & $80-160$ & $20-100$ & 6991 & 86650 & 14874 \\
174 & $110-125$ & $50-150$ & $20-100$ & 11800 & 120975 & 17562 \\
196 & $100-130$ & $50-150$ & $20-100$ & 16331 & 171483 & 17937 \\
218 & $100-130$ & $50-150$ & $20-100$ & 16113 & 171483 & 16948 \\
240 & $100-130$ & $50-150$ & $50-100$ & 15009 & 14656 & 14504 \\
262 & $100-130$ & $70-150$ & $60-100$ & 12151 & 126722 & 7016 \\
306 & $100-130$ & $110-150$ & $50-150$ & 6529 & 160592 & 2636 \\
345 & $100-130$ & $120-160$ & $20-150$ & 331 & 163809 & 183 \\
\hline
\end{tabular}

Table 6. List of kinematic cuts for the extraction of the sensitivity in figure 8 for $\sqrt{s}=350 \mathrm{GeV}$. The number of SM background events, $N_{B}^{\mathrm{SM}}$, after application of the "standard cuts" is 359500 . 


\begin{tabular}{|c|c|c|c|c|}
\hline$M[\mathrm{GeV}]$ & $P_{j j}[\mathrm{GeV}]$ & $E_{T}[\mathrm{GeV}]$ & $N_{S}$ & $N_{B}$ \\
\hline 140 & $>170$ & $<100$ & 6248 & 7550 \\
179 & $>100$ & $<100$ & 25176 & 29453 \\
218 & - & - & 43304 & 101672 \\
257 & - & - & 44691 & 101672 \\
296 & - & $50-200$ & 37571 & 65326 \\
335 & - & $70-180$ & 30710 & 44572 \\
374 & - & $90-180$ & 21766 & 29854 \\
413 & $160-220$ & - & 14926 & 20541 \\
452 & $170-230$ & - & 8551 & 15643 \\
495 & $>220$ & - & 845 & 9533 \\
\hline
\end{tabular}

Table 7. List of kinematic cuts for the extraction of the sensitivity in figure 8. For all benchmark points for the heavy neutrino mass $M$ at $\sqrt{s}=500 \mathrm{GeV}$, the pre-selection cuts have been slightly loosened to $100 \mathrm{GeV} \leq M_{j j} \leq 140 \mathrm{GeV}$.

\begin{tabular}{|c|c|c|c|}
\hline $\mathrm{M}[\mathrm{GeV}]$ & $\sqrt{s}=240 \mathrm{GeV}$ & $\sqrt{s}=350 \mathrm{GeV}$ & $\sqrt{s}=500 \mathrm{GeV}$ \\
\hline 128 & $P>86$ & $P>152$ & $P>234$ \\
150 & $P>70$ & $P>148$ & $158 \leq P \leq 224$ \\
200 & $P>70$ & $P<134$ & $64 \leq P \leq 236$ \\
300 & - & $P>152$ & $54 \leq P \leq 236$ \\
\hline
\end{tabular}

Table 8. Cuts on the Higgs boson momentum at the parton level, for selected values of the heavy neutrino mass. The cuts are chosen to maximise the significance of the signal. All momenta are in $\mathrm{GeV}$.

Open Access. This article is distributed under the terms of the Creative Commons Attribution License (CC-BY 4.0), which permits any use, distribution and reproduction in any medium, provided the original author(s) and source are credited.

\section{References}

[1] Particle Data Group collaboration, K.A. Olive et al., Review of particle physics, Chin. Phys. C 38 (2014) 090001 [INSPIRE].

[2] S. Gariazzo, C. Giunti, M. Laveder, Y.F. Li and E.M. Zavanin, Light sterile neutrinos, J. Phys. G 43 (2016) 033001 [arXiv: 1507.08204] [InSPIRE].

[3] D. Wyler and L. Wolfenstein, Massless neutrinos in left-right symmetric models, Nucl. Phys. B 218 (1983) 205 [InSPIRE].

[4] R.N. Mohapatra and J.W.F. Valle, Neutrino mass and baryon number nonconservation in superstring models, Phys. Rev. D 34 (1986) 1642 [INSPIRE].

[5] M. Shaposhnikov, A possible symmetry of the $\nu$ MSM, Nucl. Phys. B 763 (2007) 49 [hep-ph/0605047] [INSPIRE].

[6] J. Kersten and A. Yu. Smirnov, Right-handed neutrinos at CERN LHC and the mechanism of neutrino mass generation, Phys. Rev. D 76 (2007) 073005 [arXiv:0705.3221] [INSPIRE]. 
[7] M.B. Gavela, T. Hambye, D. Hernandez and P. Hernández, Minimal flavour seesaw models, JHEP 09 (2009) 038 [arXiv:0906.1461] [INSPIRE].

[8] M. Malinsky, J.C. Romao and J.W.F. Valle, Novel supersymmetric $\mathrm{SO}(10)$ seesaw mechanism, Phys. Rev. Lett. 95 (2005) 161801 [hep-ph/0506296] [INSPIRE].

[9] S. Antusch and O. Fischer, Testing sterile neutrino extensions of the Standard Model at future lepton colliders, JHEP 05 (2015) 053 [arXiv: 1502.05915] [INSPIRE].

[10] MEG collaboration, J. Adam et al., New constraint on the existence of the $\mu^{+} \rightarrow e^{+} \gamma$ decay, Phys. Rev. Lett. 110 (2013) 201801 [arXiv:1303.0754] [inSPIRE].

[11] DELPHI collaboration, P. Abreu et al., Search for neutral heavy leptons produced in $Z$ decays, Z. Phys. C 74 (1997) 57 [Erratum ibid. C 75 (1997) 580] [INSPIRE].

[12] OPAL collaboration, M.Z. Akrawy et al., Limits on neutral heavy lepton production from $Z^{0}$ decay, Phys. Lett. B 247 (1990) 448 [inSPIRE].

[13] ALEPH collaboration, D. Decamp et al., Searches for new particles in $Z$ decays using the ALEPH detector, Phys. Rept. 216 (1992) 253 [INSPIRE].

[14] L3 collaboration, O. Adriani et al., Results from the L3 experiment at LEP, Phys. Rept. 236 (1993) 1 [INSPIRE].

[15] F. del Aguila and J.A. Aguilar-Saavedra, Electroweak scale seesaw and heavy Dirac neutrino signals at LHC, Phys. Lett. B 672 (2009) 158 [arXiv:0809.2096] [InSPIRE].

[16] F. del Aguila, J.A. Aguilar-Saavedra and J. de Blas, Trilepton signals: the golden channel for seesaw searches at LHC, Acta Phys. Polon. B 40 (2009) 2901 [arXiv:0910.2720] [inSPIRE].

[17] A. Das and N. Okada, Inverse seesaw neutrino signatures at the LHC and ILC, Phys. Rev. D 88 (2013) 113001 [arXiv: 1207.3734] [INSPIRE].

[18] K.N. Abazajian et al., Light sterile neutrinos: a white paper, arXiv:1204.5379 [INSPIRE].

[19] P.S. Bhupal Dev, R. Franceschini and R.N. Mohapatra, Bounds on TeV seesaw models from LHC Higgs data, Phys. Rev. D 86 (2012) 093010 [arXiv:1207.2756] [INSPIRE].

[20] M. Drewes, The phenomenology of right handed neutrinos, Int. J. Mod. Phys. E 22 (2013) 1330019 [arXiv: 1303.6912] [INSPIRE].

[21] C.-H. Lee, P.S. Bhupal Dev and R.N. Mohapatra, Natural TeV-scale left-right seesaw mechanism for neutrinos and experimental tests, Phys. Rev. D 88 (2013) 093010 [arXiv: 1309.0774] [INSPIRE].

[22] J.C. Helo, M. Hirsch and S. Kovalenko, Heavy neutrino searches at the LHC with displaced vertices, Phys. Rev. D 89 (2014) 073005 [arXiv: 1312.2900] [INSPIRE].

[23] A. Das, P.S. Bhupal Dev and N. Okada, Direct bounds on electroweak scale pseudo-Dirac neutrinos from $\sqrt{s}=8 \mathrm{TeV}$ LHC data, Phys. Lett. B 735 (2014) 364 [arXiv:1405.0177] [INSPIRE].

[24] FCC-Ee study Team collaboration, A. Blondel, E. Graverini, N. Serra and M. Shaposhnikov, Search for heavy right handed neutrinos at the FCC-ee, arXiv:1411.5230 [INSPIRE].

[25] A. Abada, V. De Romeri, S. Monteil, J. Orloff and A.M. Teixeira, Indirect searches for sterile neutrinos at a high-luminosity Z-factory, JHEP 04 (2015) 051 [arXiv:1412.6322] [INSPIRE]. 
[26] M. Drewes and B. Garbrecht, Experimental and cosmological constraints on heavy neutrinos, arXiv: 1502.00477 [INSPIRE].

[27] F.F. Deppisch, P.S. Bhupal Dev and A. Pilaftsis, Neutrinos and collider physics, New J. Phys. 17 (2015) 075019 [arXiv: 1502.06541] [INSPIRE].

[28] P. Humbert, M. Lindner and J. Smirnov, The inverse seesaw in conformal electro-weak symmetry breaking and phenomenological consequences, JHEP 06 (2015) 035 [arXiv: 1503.03066] [INSPIRE].

[29] S. Banerjee, P.S.B. Dev, A. Ibarra, T. Mandal and M. Mitra, Prospects of heavy neutrino searches at future lepton colliders, Phys. Rev. D 92 (2015) 075002 [arXiv:1503.05491] [INSPIRE].

[30] S. Antusch and O. Fischer, Testing sterile neutrino extensions of the Standard Model at the circular electron positron collider, Int. J. Mod. Phys. A 30 (2015) 1544004 [inSPIRE].

[31] L. Duarte, J. Peressutti and O.A. Sampayo, Majorana neutrino decay in an effective approach, Phys. Rev. D 92 (2015) 093002 [arXiv: 1508.01588] [INSPIRE].

[32] N. Bizot and M. Frigerio, Fermionic extensions of the Standard Model in light of the Higgs couplings, JHEP 01 (2016) 036 [arXiv:1508.01645] [INSPIRE].

[33] C.O. Dib and C.S. Kim, Discovering sterile neutrinos ligther than $M_{W}$ at the LHC, Phys. Rev. D 92 (2015) 093009 [arXiv: 1509.05981] [INSPIRE].

[34] A. Das and N. Okada, Improved bounds on the heavy neutrino productions at the LHC, Phys. Rev. D 93 (2016) 033003 [arXiv:1510.04790] [InSPIRE].

[35] A. Abada, V. De Romeri and A.M. Teixeira, Impact of sterile neutrinos on nuclear-assisted cLFV processes, JHEP 02 (2016) 083 [arXiv: 1510.06657] [INSPIRE].

[36] A. de Gouvêa and A. Kobach, Global constraints on a heavy neutrino, Phys. Rev. D 93 (2016) 033005 [arXiv: 1511.00683] [INSPIRE].

[37] L. Basso, Resonant mono Higgs at the LHC, JHEP 04 (2016) 087 [arXiv:1512.06381] [INSPIRE].

[38] A. Datta, M. Guchait and A. Pilaftsis, Probing lepton number violation via Majorana neutrinos at hadron supercolliders, Phys. Rev. D 50 (1994) 3195 [hep-ph/9311257] [INSPIRE].

[39] T. Han and B. Zhang, Signatures for Majorana neutrinos at hadron colliders, Phys. Rev. Lett. 97 (2006) 171804 [hep-ph/0604064] [INSPIRE].

[40] C.-Y. Chen and P.S.B. Dev, Multi-lepton collider signatures of heavy Dirac and Majorana neutrinos, Phys. Rev. D 85 (2012) 093018 [arXiv:1112.6419] [INSPIRE].

[41] P.S.B. Dev, A. Pilaftsis and U.-K. Yang, New production mechanism for heavy neutrinos at the LHC, Phys. Rev. Lett. 112 (2014) 081801 [arXiv: 1308.2209] [InSPIRE].

[42] D. Alva, T. Han and R. Ruiz, Heavy Majorana neutrinos from $W \gamma$ fusion at hadron colliders, JHEP 02 (2015) 072 [arXiv:1411.7305] [INSPIRE].

[43] F.F. Deppisch, P.S. Bhupal Dev and A. Pilaftsis, Neutrinos and collider physics, New J. Phys. 17 (2015) 075019 [arXiv: 1502.06541] [INSPIRE].

[44] S. Banerjee, P.S.B. Dev, A. Ibarra, T. Mandal and M. Mitra, Prospects of heavy neutrino searches at future lepton colliders, Phys. Rev. D 92 (2015) 075002 [arXiv:1503.05491] [INSPIRE]. 
[45] C.O. Dib and C.S. Kim, Discovering sterile neutrinos ligther than $M_{W}$ at the LHC, Phys. Rev. D 92 (2015) 093009 [arXiv: 1509.05981] [INSPIRE].

[46] S. Antusch, C. Biggio, E. Fernandez-Martinez, M.B. Gavela and J. Lopez-Pavon, Unitarity of the leptonic mixing matrix, JHEP 10 (2006) 084 [hep-ph/0607020] [INSPIRE].

[47] S. Antusch and O. Fischer, Non-unitarity of the leptonic mixing matrix: present bounds and future sensitivities, JHEP 10 (2014) 094 [arXiv: 1407.6607] [INSPIRE].

[48] TLeP Design Study Working Group collaboration, M. Bicer et al., First look at the physics case of TLEP, JHEP 01 (2014) 164 [arXiv:1308.6176] [INSPIRE].

[49] M. Ruan, Higgs measurement at $e^{+} e^{-}$circular colliders, arXiv:1411.5606 [INSPIRE].

[50] R. Tenchini, Precision electroweak measurements at FCC-ee, arXiv:1412.2928 [INSPIRE].

[51] H. Baer et al., The International Linear Collider technical design report - volume 2: physics, arXiv:1306.6352 [INSPIRE].

[52] W. Kilian, M. Krämer and P.M. Zerwas, Higgsstrahlung and $W W$ fusion in $e^{+} e^{-}$collisions, Phys. Lett. B 373 (1996) 135 [hep-ph/9512355] [INSPIRE].

[53] W. Kilian, T. Ohl and J. Reuter, WHIZARD: simulating multi-particle processes at LHC and ILC, Eur. Phys. J. C 71 (2011) 1742 [arXiv:0708.4233] [INSPIRE].

[54] M. Moretti, T. Ohl and J. Reuter, O'Mega: an optimizing matrix element generator, hep-ph/0102195 [INSPIRE].

[55] W. Buchmüller and C. Greub, Heavy Majorana neutrinos in electron-positron and electron-proton collisions, Nucl. Phys. B 363 (1991) 345 [INSPIRE].

[56] D. Neuffer, M. Palmer, Y. Alexahin, C. Ankenbrandt and J.P. Delahaye, A muon collider as a Higgs factory, arXiv: 1502.02042 [INSPIRE].

[57] E. Fernandez-Martinez, J. Hernandez-Garcia, J. Lopez-Pavon and M. Lucente, Loop level constraints on seesaw neutrino mixing, JHEP 10 (2015) 130 [arXiv:1508.03051] [INSPIRE].

[58] A. Alloul, N.D. Christensen, C. Degrande, C. Duhr and B. Fuks, FeynRules $2.0-a$ complete toolbox for tree-level phenomenology, Comput. Phys. Commun. 185 (2014) 2250 [arXiv:1310.1921] [INSPIRE].

[59] J. Alwall et al., The automated computation of tree-level and next-to-leading order differential cross sections and their matching to parton shower simulations, JHEP 07 (2014) 079 [arXiv: 1405.0301] [INSPIRE].

[60] E. Conte, B. Fuks and G. Serret, MadAnalysis 5, a user-friendly framework for collider phenomenology, Comput. Phys. Commun. 184 (2013) 222 [arXiv:1206.1599] [INSPIRE].

[61] T. Sjöstrand, S. Mrenna and P.Z. Skands, PYTHIA 6.4 physics and manual, JHEP 05 (2006) 026 [hep-ph/0603175] [INSPIRE].

[62] M. Cacciari, G.P. Salam and G. Soyez, FastJet user manual, Eur. Phys. J. C 72 (2012) 1896 [arXiv:1111.6097] [INSPIRE].

[63] H. Ono and A. Miyamoto, A study of measurement precision of the Higgs boson branching ratios at the International Linear Collider, Eur. Phys. J. C 73 (2013) 2343 [arXiv: 1207.0300] [INSPIRE].

[64] L. Basso, O. Fischer and J.J. van der Bij, Precision tests of unitarity in leptonic mixing, Europhys. Lett. 105 (2014) 11001 [arXiv:1310.2057] [INSPIRE]. 